\title{
Dynamical Behaviors of Stochastic Delayed One-Predator and Two-Competing-Prey Systems with Holling Type IV and Crowley-Martin Type Functional Responses
}

\author{
Changjian Wang, Zuoliang Xiong, Rensheng He, and Hongwei Yin \\ Department of Mathematics, Nanchang University, Nanchang, Jiangxi 330031, China \\ Correspondence should be addressed to Zuoliang Xiong; xiong1601@163.com
}

Received 6 July 2016; Accepted 13 October 2016

Academic Editor: Douglas R. Anderson

Copyright (C) 2016 Changjian Wang et al. This is an open access article distributed under the Creative Commons Attribution License, which permits unrestricted use, distribution, and reproduction in any medium, provided the original work is properly cited.

\begin{abstract}
This paper is devoted to stochastic delayed one-predator and two-competing-prey systems with two kinds of different functional responses. By establishing appropriate Lyapunov functions, the globally positive solution and stochastic boundedness are investigated. In some case, the stochastic permanence and extinction are also obtained. Moreover, sufficient conditions of the global asymptotic stability of the system are established. Finally, some numerical examples are provided to explain our conclusions.
\end{abstract}

\section{Introduction}

It is well known that predator-prey system, cooperative system, and competitive system are three kinds of important ecological systems. The dynamic relationship among species is a significant theme whether in ecology or in mathematical ecology because of its importance and universal existence with many concerned biological systems (see [1]). A lot of systems about predator-prey behaviors have been proposed (see [2-4]).

A main objective for ecologists is to find the relationships among species. And the consumption rate of each predator on prey is an important component of the relationships between predator and prey, that is, predator's functional response. In order to describe different situations when predators search or compete for food, many significant functional responses have been proposed, such as L-V and Holling II-IV types (see [5-7]). A suitable functional response is not only related to the density of prey, but also to the predator. A statistics from 19 predator-prey systems indicates that CrowleyMartin type, Beddington-DeAngelis type, and Hassell-Varley type predator-dependent functions can provide a better description in some case. In [8], the following predator-prey system bas been studied:

$$
\begin{aligned}
& \frac{d x}{d t}=x\left(A-B x-\frac{C y}{A_{1}+B_{1} x+C_{1} y+B_{1} C_{1} x y}\right), \\
& \frac{d y}{d t}=y\left(-D-E y+\frac{F x}{A_{1}+B_{1} x+C_{1} y+B_{1} C_{1} x y}\right),
\end{aligned}
$$

where $x$ is the density of prey and $y$ is the density of predator at time $t$.

However, interaction of multiple species often occurs in nature and their relationships are much more complex than that of the two species (see $[9,10])$. Therefore, it is more realistic to study the multiple species predator-prey systems. Motivated by above, we consider the following systems:

$$
\begin{aligned}
d y_{1} & =y_{1}\left(a_{1}-b_{1} y_{1}-\frac{c_{2} y_{2}}{1+y_{2}}\right. \\
& \left.-\frac{c_{3} z}{1+m y_{1}+n z+k y_{1} z}\right) d t,
\end{aligned}
$$




$$
\begin{aligned}
d y_{2} & =y_{2}\left(a_{2}-b_{2} y_{2}-\frac{c_{1} y_{1}}{1+y_{1}}-\frac{c_{4} z}{1+m_{1} y_{2}^{2}}\right) d t \\
d z & =y\left(a_{3}-b_{3} z+\frac{d_{1} y_{1}}{1+m y_{1}+n z+k y_{1} z}\right. \\
& \left.+\frac{d_{2} y_{2}}{1+m_{1} y_{2}^{2}}\right) d t
\end{aligned}
$$

where $y_{1}, y_{2}$ and $z$ denote two competed prey and predator densities, respectively. And the parameters $a_{1}, a_{2}$, and $a_{3}$ are the intrinsic growth of three species; $b_{1}, b_{2}$, and $b_{3}$ are the intraspecific competition rate of three species, respectively. $c_{1}$ and $c_{2}$ are the interspecific competition rates of two competed species, $c_{3}$ and $c_{4}$ are the predators' capturing rates, and $d_{1}$ and $d_{2}$ are the rates of conversion of nutrients into the production of predator. And all the parameters in system (2) are constants. It is very necessary to point out that $c_{3} z /(1+$ $\left.m y_{1}+n z+k y_{1} z\right)$ is a special functional response; when $m=$ $n=k=0$ it becomes a linear mass-action function response (or Holling type I functional response), when $n=k=0$ it becomes a Holling type II functional response, when $k=0$ it becomes a modified Holling type II functional response, and when $m n=k$ it becomes a Crowley-Martin functional response.

In the real world, population dynamics are often affected by white noise from the environment, which relate to climate, geographical distribution, geological features, human disaster, human intervention, and other environmental factors. Therefore, the flow of biological energy is a process of fluctuation. The oscillation of population biomass is directly related to the birth and death rate of random perturbation. Up to now, there have been many works considering the effect of random perturbation (see [11-13]). In this paper, we assume that white noise affects the intrinsic birth rate, capture rate of predator, and conversion rate of the predator population. On the other hand, the development trend of the real biological system is not only related to the status of the system, but also depends on the history of the system more or less, which is called time delays. Moreover, time delay widely exists in biological systems; for example, in the predator-prey system, the process for the conversion of prey to predators is not immediately translated into the predator population but after a certain period of time to digest the transformation. So a more realistic predator-prey model should consider the effects of time delays (see $[14,15]$ ). As a matter of fact, delay differential systems have much more complicated dynamical behaviors than the differential equations without delays. Therefore, the following stochastic delay systems are considered:

$$
\begin{aligned}
d y_{1} & =y_{1}\left[a_{1}-b_{1} y_{1}-\frac{c_{2} y_{2}\left(t-\tau_{2}\right)}{1+y_{2}\left(t-\tau_{2}\right)}\right. \\
& \left.-\frac{c_{3} z}{1+m y_{1}+n z+k y_{1} z}\right] d t+\sigma_{1} y_{1} d B_{1}(t)
\end{aligned}
$$

$$
\begin{aligned}
& +\frac{\sigma_{2} y_{1} z}{1+m y_{1}+n z+k y_{1} z} d B_{2}(t) \\
d y_{2} & =y_{2}\left[a_{2}-b_{2} y_{2}-\frac{c_{1} y_{1}\left(t-\tau_{1}\right)}{1+y_{1}\left(t-\tau_{1}\right)}-\frac{c_{4} z}{1+m_{1} y_{2}^{2}}\right] d t \\
& +\sigma_{3} y_{2} d B_{3}(t), \\
d z & =z\left[a_{3}-b_{3} z\right. \\
& +\frac{d y_{1}\left(t-\tau_{3}\right)}{1+m y_{1}\left(t-\tau_{3}\right)+n z\left(t-\tau_{3}\right)+k y_{1}\left(t-\tau_{3}\right) z\left(t-\tau_{3}\right)} \\
& \left.+\frac{d_{2} y_{2}\left(t-\tau_{3}\right)}{1+m_{1} y_{2}^{2}\left(t-\tau_{3}\right)}\right] d t+\sigma_{4} z d B_{4}(t) \\
& +\frac{\sigma_{5} y_{1} z}{1+m y_{1}+n z+k y_{1} z} d B_{5}(t) .
\end{aligned}
$$

$\sigma_{i}^{2}$ are the intensities of the noises, $i=1,2,3,4,5 . B_{i}(t)$ are standard Brownian motions which are defined on a complete probability space $(\Omega, F, P), i=1,2,3,4,5$. Let $\tau=\max \left\{\tau_{1}, \tau_{2}, \tau_{3}\right\}$ and $C=C\left([-\tau, 0], R_{+}^{3}\right)$ be the set of continuous functions from $[-\tau, 0]$ to $R_{+}^{3}$ with initial condition $\gamma \in C\left([-\tau, 0], R_{+}^{3}\right)$ and the norm $\|\gamma\|=\sup _{-\tau \leq \theta \leq 0} \xi(\theta)<$ $+\infty$.

Any biological system, whether it is population, biological communities, ecosystems, or the biosphere, its dynamic behavior is one of the main objects of the study, such as the resistance of ecosystem, persistence, recoverability, variability, and consistency. Therefore, we use mathematical theory and methods to study the dynamics of biological populations, which can not only protect the ecological balance but also can improve the ecological environment for human survival. According to what we know, few current literatures are found to discuss stochastic delayed predator-prey systems with Holling type IV and Crowley-Martin type functional responses at the same time. In this paper, it is the first time to obtain the condition of global asymptotic stability of system (3).

This paper is carried out as follows. In Section 2 and in Section 3, global positive solution and stochastically ultimate boundedness of system (3) are investigated. In Sections 4 and 5, we study the stochastic permanence and extinction, respectively. In Section 6, we obtain the fact that system (3) is globally asymptotically stable. In the end, in Section 7 , some numerical examples are provided to explain our findings.

\section{Existence of Global Positive Solution}

Theorem 1. For arbitrary initial data $\gamma \in C\left([-\tau, 0], R_{+}^{3}\right)$, system (3) has a unique positive solution on $\tau \geq 0$ which will remain in $R_{+}^{3}$ with probability 1 . 
Proof. Define a $C^{3}$-function $V_{1} \rightarrow R_{+}$by

$$
\begin{aligned}
V_{1}\left(y_{1}, y_{2}, z\right)= & y_{1}-1-\ln y_{1}+y_{2}-1-\ln y_{2}+z-1 \\
& -\ln z .
\end{aligned}
$$

Clearly, $V_{1}\left(y_{1}, y_{2}, z\right)$ is nonnegative when $y_{1}>0, y_{2}>$ $0, z>0$. Now we continue to define

$$
\begin{aligned}
V_{2}\left(y_{1}, y_{2}, z\right)= & V_{1}\left(y_{1}, y_{2}, z\right)+c_{1} \int_{t-\tau_{1}}^{t} y_{1}(s) d s \\
& +c_{2} \int_{t-\tau_{2}}^{t} y_{2}(s) d s,
\end{aligned}
$$

where $c_{1}, c_{2}$ are the same in system (3). By the same method in [13], we can complete the proof and omit it here.

\section{Stochastically Ultimate Boundedness}

Stochastically ultimate boundedness of system (3) is studied in this part. Firstly, we present a useful lemma in the following.

Lemma 2. For any initial data $\gamma \in C\left([-\tau, 0], R_{+}^{3}\right),\left(y_{1}(t)\right.$, $\left.y_{2}(t), z(t)\right)$ is a positive solution of system (3); there exist three positive constants $K_{1}(p), K_{2}(p)$, and $K_{3}(p), p>1$, which satisfy

$$
\begin{gathered}
\lim _{t \rightarrow \infty} \mathbf{E}\left|y_{1}(t)\right|^{p}=K_{1}(p), \\
\lim _{t \rightarrow \infty} \mathbf{E}\left|y_{2}(t)\right|^{p}=K_{2}(p), \\
\lim _{t \rightarrow \infty} \mathbf{E}|z(t)|^{p}=K_{3}(p) .
\end{gathered}
$$

Proof. We define $V_{1}\left(y_{1}\right)=y_{1}^{p}, V_{2}\left(y_{2}\right)=y_{2}^{p}$, and $V_{3}(z)=z^{p}$. By Itô's formula, we have

$$
\begin{aligned}
d V_{1} & =p y_{1}^{p-1} d y_{1}+\frac{1}{2} p(p-1) y_{1}^{p-2}\left(d y_{1}\right)^{2} \\
& =p y_{1}^{p} d y_{1}\left[a_{1}-b_{1} y_{1}-\frac{c_{2} y_{2}\left(t-\tau_{2}\right)}{1+y_{2}\left(t-\tau_{2}\right)}\right. \\
& -\frac{c_{3} z}{1+m y_{1}+n z+k y_{1} z}+\frac{1}{2}(p-1) \\
& \left.+\left(\sigma_{1}^{2}+\frac{\sigma_{2}^{2} z^{2}}{2\left(1+m y_{1}+n z+k y_{1} z\right)^{2}}\right)\right] d t \\
& +\sigma_{1} p y_{1}^{p} d B_{1}(t)+\frac{\sigma_{2} p y_{1}^{p} z}{\left(1+m y_{1}+n z+k y_{1} z\right)} d B_{2}(t) \\
& \leqslant p y_{1}^{p}\left[a_{1}-b_{1} y_{1}+\frac{1}{2}(p-1)+\frac{(p-1) \sigma_{2}^{2}}{2 n_{2}}\right] \\
+ & \sigma_{1} p y_{1}^{p} d B_{1}(t)+\frac{\sigma_{2} p y_{1}^{p} z}{\left(1+m y_{1}+n z+k y_{1} z\right)} d B_{2}(t) .
\end{aligned}
$$

Also, we can obtain that

$$
\begin{aligned}
& d V_{2}=p y_{2}^{p-1} d y_{2}+\frac{1}{2} p(p-1) y_{2}^{p-2}\left(d y_{2}\right)^{2}=p y_{2}^{p}\left[a_{2}\right. \\
& \left.\quad-b_{2} y_{2}-\frac{c_{1} y_{1}\left(t-\tau_{1}\right)}{1+y_{1}\left(t-\tau_{1}\right)}-\frac{c_{4} z}{1+m_{1} y_{2}^{2}}\right]+\sigma_{3} p y_{2}^{p} d B_{3}(t) \\
& \quad \leqslant p y_{2}^{p}\left[a_{2}-b_{2} y_{2}+\frac{1}{2}(p-1) \sigma_{3}^{2}\right] d t+p y_{2}^{p} \sigma_{3} d B_{3}(t) \\
& d V_{3}=p z^{p-1} d z+\frac{1}{2} p(p-1) z^{p-2}(d y)^{2}=p z^{p}\left[a_{3}-b_{3} z\right. \\
& +\frac{d_{1} y_{1}\left(t-\tau_{3}\right)}{1+m y_{1}\left(t-\tau_{3}\right)+n z\left(t-\tau_{3}\right)+k y_{1}\left(t-\tau_{3}\right) z\left(t-\tau_{3}\right)} \\
& +\frac{d_{2} y_{2}\left(t-\tau_{3}\right)}{1+m_{1} y_{2}^{2}\left(t-\tau_{3}\right)} \\
& +\frac{\sigma_{5} y_{1} z^{p}}{1+m y_{1}+n z+k y_{1} z} d B_{5}(t) . \\
& +\frac{1}{2}(p-1)\left(\sigma_{4} p y^{p} d B_{4}(t)+\frac{\sigma_{5} x_{1} y^{p}}{1+m x_{1}+n z+k x_{1} z} d B_{5}(t)\right. \\
& +a_{1}+\frac{d_{2}^{2}}{2}+\frac{1}{2 m_{1}}-b_{3} z \\
& +\sigma_{5}^{2} y_{1}^{2} \\
& \left.\left.\left.+m y_{1}+n z+k y_{1} z\right)^{2}\right)\right] d t
\end{aligned}
$$

By taking expectation in both sides of inequality (9), we have that

$$
\begin{aligned}
& \frac{d \mathbf{E}\left[y_{1}^{p}(t)\right]}{d t} \leqslant p\left[a_{1}+\frac{(p-1) \sigma_{1}^{2}}{2}+\frac{(p-1) \sigma_{2}^{2}}{2 n^{2}}\right] \\
& \cdot \mathbf{E}\left[y_{1}^{p}(t)\right]-b_{1} p \mathbf{E}\left[y_{1}^{p+1}(t)\right] \leqslant p\left[a_{1}\right. \\
& \left.+\frac{(p-1) \sigma_{1}^{2}}{2}+\frac{(p-1) \sigma_{2}^{2}}{2 n^{2}}\right] \mathbf{E}\left[y_{1}^{p}(t)\right] \\
& -b_{1} p\left[\mathbf{E}\left(y_{1}^{p}(t)\right)\right]^{(p+1) / p} \leqslant p \mathbf{E}\left[y_{1}^{p}(t)\right]\left\{a_{1}\right. \\
& \left.+\frac{(p-1) \sigma_{1}^{2}}{2}+\frac{(p-1) \sigma_{2}^{2}}{2 n^{2}}-b_{1}\left[\mathbf{E}\left(y_{1}^{p}(t)\right)\right]^{1 / p}\right\} .
\end{aligned}
$$


By the same way, we can obtain that

$$
\begin{aligned}
& \frac{d \mathbf{E}\left[y_{2}^{p}(t)\right]}{d t} \leqslant p \mathbf{E}\left[y_{2}^{p}(t)\right]\left\{a_{2}+\frac{(p-1) \sigma_{3}^{2}}{2}\right. \\
& \left.-b_{2}\left[\mathbf{E}\left(y_{2}^{p}(t)\right)\right]^{1 / p}\right\}, \\
& \frac{d \mathbf{E}\left[z^{p}(t)\right]}{d t} \leqslant p \mathbf{E}\left[z^{p}(t)\right]\left\{a_{3}+\frac{d_{1}}{m}+\frac{d_{2}^{2}}{2}+\frac{1}{2 m_{1}}\right. \\
& \left.+\frac{1}{2}(p-1)\left(\sigma_{4}^{2}+\frac{\sigma_{5}^{2}}{m^{2}}\right)-b_{3}\left[\mathbf{E}\left(z^{p}(t)\right)\right]^{1 / p}\right\} .
\end{aligned}
$$

$$
\begin{aligned}
& K_{1}(p)=\left(\frac{a_{1}+(p-1) \sigma_{1}^{2} / 2+(p-1) \sigma_{2}^{2} / 2 n^{2}}{b_{1}}\right)^{p}, \\
& K_{2}(p)=\left(\frac{a_{2}+(p-1) \sigma_{3}^{2} / 2}{b_{2}}\right)^{p}, \\
& K_{3}(p)=\left(\frac{a_{3}+d_{1} / m+d_{2}^{2} / 2+1 / 2 m_{1}+(1 / 2)(p-1)\left(\sigma_{4}^{2}+\sigma_{5}^{2} / m^{2}\right)}{b_{3}}\right)^{p} .
\end{aligned}
$$

Therefore, we have

$$
\begin{aligned}
& \lim _{n \rightarrow \infty} \mathbf{E}\left|y_{1}(t)\right|^{p}=K_{1}(p), \\
& \lim _{n \rightarrow \infty} \mathbf{E}\left|y_{2}(t)\right|^{p}=K_{2}(p), \\
& \lim _{n \rightarrow \infty} \mathbf{E}|z(t)|^{p}=K_{3}(p),
\end{aligned}
$$

where
This completes the proof.

Theorem 3. For arbitrary initial data $\gamma \in C\left([-\tau, 0], R_{+}^{3}\right)$, by the definition of stochastic boundedness, one has the fact that system (3) is stochastically ultimately bounded.

Proof. From Lemma 2, we can see that $\mathbf{E}\left[\mid y_{1}(t), y_{2}(t)\right.$, $\left.\left.z(t)\right|^{p}\right] \leqslant K(p)$. For any $\epsilon \in(0,1)$, let $G=[K(p) / \epsilon]^{1 / p}, p>1$; then using Chebyshev inequality, we can obtain that

$$
\begin{aligned}
P\left\{\left|y_{1}(t), y_{2}(t), z(t)\right|>G\right\} & \\
< & \frac{\mathbf{E}\left[\left|y_{1}(t), y_{2}(t), z(t)\right|^{p}\right]}{G^{p}} \leqslant \epsilon,
\end{aligned}
$$

where $K(p)=3^{p / 2}\left(K_{1}(p)+K_{2}(p)+K_{3}(p)\right)$.

This completes the proof of Theorem 3 .

\section{Stochastic Permanence}

Theorem 4. If

$$
\begin{gathered}
\frac{1}{2} \max \left\{\sigma_{1}^{2}+\frac{\sigma_{2}^{2}+\sigma_{5}^{2}}{n^{2}}, \sigma_{3}^{2}, \sigma_{4}^{2}+\frac{\sigma_{2}^{2}+\sigma_{5}^{2}}{m^{2}}\right\} \\
<\min \left\{a_{1}-c_{2}, a_{2}-c_{1}, a_{3}-\frac{c_{3}}{m}\right\}
\end{gathered}
$$

holds, by the definition of stochastic permanence, we say that system (3) is stochastically permanent. 


$$
\begin{aligned}
& \left.-b_{2} y_{2}-\frac{c_{1} y_{1}\left(t-\tau_{1}\right)}{1+y_{1}\left(t-\tau_{1}\right)}-\frac{c_{4} z}{1+m_{1} y_{2}^{2}}\right]+z\left[a_{3}-b_{3} z\right. \\
& +\frac{d_{1} y_{1}\left(t-\tau_{3}\right)}{1+m y_{1}\left(t-\tau_{3}\right)+n z\left(t-\tau_{3}\right)+k x_{1}\left(t-\tau_{3}\right) z\left(t-\tau_{3}\right)} \\
& \left.\left.+\frac{d_{2} y_{2}\left(t-\tau_{3}\right)}{1+m_{1} y_{2}^{2}\left(t-\tau_{3}\right)}\right]\right\} d t+W^{3}\left[\sigma_{1}^{2} y_{1}^{2}+\sigma_{3}^{2} y_{2}^{2}\right. \\
& +\sigma_{4}^{2} z^{2}+\frac{\sigma_{2}^{2} y_{1}^{2} z^{2}}{\left(1+m y_{1}+n z+k y_{1} z\right)^{2}} \\
& \left.+\frac{\sigma_{5}^{2} y_{1}^{2} z^{2}}{\left(1+m y_{1}+n z+k y_{1} z\right)^{2}}\right] d t-W^{2}\left[\sigma_{1} y_{1} d B_{1}(t)\right. \\
& +\sigma_{3} y_{2} d B_{3}(t)+\sigma_{4} z d B_{4}(t) \\
& \left.+\frac{y_{1} z}{1+m y_{1}+n z+k y_{1} z}\left(\sigma_{2} d B_{2}(t)+\sigma_{5} d B_{5}(t)\right)\right] \\
& \left.+\frac{y_{1} z}{1+m y_{1}+n z+k y_{1} z}\left(\sigma_{2} d B_{2}(t)+\sigma_{5} d B_{5}(t)\right)\right] \\
& +L W d t-W^{2}\left[\sigma_{1} y_{1} d B_{1}(t)+\sigma_{3} y_{2} d B_{3}(t)+\sigma_{4} z d B_{4}(t)\right.
\end{aligned}
$$

where

$$
\begin{aligned}
& L W=-W^{2}\left\{y _ { 1 } \left[a_{1}-b_{1} y_{1}-\frac{c_{2} y_{2}\left(t-\tau_{2}\right)}{1+y_{2}\left(t-\tau_{2}\right)}\right.\right. \\
& \left.-\frac{c_{3} z}{1+m y_{1}+n z+k y_{1} z}\right]+y_{2}\left[a_{2}-b_{2} y_{2}\right. \\
& \left.-\frac{c_{1} y_{1}\left(t-\tau_{1}\right)}{1+y_{1}\left(t-\tau_{1}\right)}-\frac{c_{4} z}{1+m_{1} y_{2}^{2}}\right]+z\left[a_{3}-b_{3} z\right. \\
& +\frac{d_{1} y_{1}\left(t-\tau_{3}\right)}{1+m y_{1}\left(t-\tau_{3}\right)+n z\left(t-\tau_{3}\right)+k x_{1}\left(t-\tau_{3}\right) z\left(t-\tau_{3}\right)} \\
& \left.\left.+\frac{d_{2} y_{2}\left(t-\tau_{3}\right)}{1+m_{1} y_{2}^{2}\left(t-\tau_{3}\right)}\right]\right\}+W^{3}\left[\sigma_{1}^{2} y_{1}^{2}+\sigma_{3}^{2} y_{2}^{2}+\sigma_{4}^{2} z^{2}\right. \\
& \left.+\frac{\left(\sigma_{2}^{2}+\sigma_{5}^{2}\right) y_{1}^{2} z^{2}}{\left(1+m y_{1}+n z+k y_{1} z\right)^{2}}\right] .
\end{aligned}
$$

If (16) holds, we can find a positive constant $\mu$ which satisfies the following condition:

$$
\begin{aligned}
& \frac{1+\mu}{2} \max \left\{\sigma_{1}^{2}+\frac{\sigma_{2}^{2}+\sigma_{5}^{2}}{n^{2}}, \sigma_{3}^{2}, \sigma_{4}^{2}+\frac{\sigma_{2}^{2}+\sigma_{5}^{2}}{m^{2}}\right\} \\
& <\min \left\{a_{1}-c_{2}, a_{2}-c_{1}, a_{3}-\frac{c_{3}}{m}\right\} .
\end{aligned}
$$

Using Itô's formula, we can obtain

$$
\begin{aligned}
& d(1+W)^{\mu}=\left[\mu(1+W)^{\mu-1} L W+\frac{\mu(\mu-1)}{2} W^{4}(1\right. \\
& +W)^{\mu-2}\left(\sigma_{1}^{2} y_{1}^{2}+\sigma_{3}^{2} y_{2}^{2}+\sigma_{4}^{2} z^{2}\right. \\
& \left.\left.+\frac{\left(\sigma_{2}^{2}+\sigma_{5}^{2}\right) y_{1}^{2} z^{2}}{\left(1+m y_{1}+n z+k y_{1} z\right)^{2}}\right)\right] d t+\mu W^{2}(1 \\
& +W)^{\mu-1}\left(\sigma_{1} y_{1} d B_{1}(t)+\sigma_{3} y_{2} d B_{3}(t)+\sigma_{4} z d B_{4}(t)\right. \\
& \left.+\frac{y_{1} z}{1+m y_{1}+n z+k y_{1} z}\left(\sigma_{2} d B_{2}(t)+\sigma_{5} d B_{5}(t)\right)\right) .
\end{aligned}
$$

Hence,

$$
\begin{aligned}
& L(1+W)^{\mu}=\mu(1+W)^{\mu-1} L W+\frac{\mu(\mu-1)}{2} W^{4}(1 \\
& +W)^{\mu-2}\left(\sigma_{1}^{2} y_{1}^{2}+\sigma_{3}^{2} y_{2}^{2}+\sigma_{4}^{2} z^{2}\right.
\end{aligned}
$$

$$
\left.+\frac{\left(\sigma_{2}^{2}+\sigma_{5}^{2}\right) y_{1}^{2} z^{2}}{\left(1+m x_{1}+n z+k x_{1} z\right)^{2}}\right)
$$

Under the condition of (20), we can choose another positive constant $k$ making it satisfy the following condition:

$$
\begin{aligned}
& \frac{\mu(1+\mu)}{2} \max \left\{\sigma_{1}^{2}+\frac{\sigma_{2}^{2}+\sigma_{5}^{2}}{n^{2}}, \sigma_{3}^{2}, \sigma_{4}^{2}+\frac{\sigma_{2}^{2}+\sigma_{5}^{2}}{m^{2}}\right\} \\
& <\min \left\{a_{1}-c_{2}, a_{2}-c_{1}, a_{3}-\frac{c_{3}}{m}\right\}-k .
\end{aligned}
$$

Using Itô's formula, we can obtain

$$
d\left[e^{k t}(1+W)^{\mu}\right]=e^{k t} d(1+W)^{\mu}+k e^{k t}(1+W)^{\mu} d t
$$

Hence,

$$
\begin{gathered}
L e^{k t}(1+W)^{\mu}=e^{k t} L W+k e^{k t}(1+W)^{\mu}+\frac{\mu(\mu-1)}{2} \\
\cdot W^{4}(1+W)^{\mu-2}\left(\sigma_{1}^{2} y_{1}^{2}+\sigma_{3}^{2} y_{2}^{2}+\sigma_{4}^{2} z^{2}\right. \\
\left.+\frac{\left(\sigma_{2}^{2}+\sigma_{5}^{2}\right) y_{1}^{2} z^{2}}{\left(1+m y_{1}+n z+k y_{1} z\right)^{2}}\right) \leqslant k e^{k t}(1+W)^{\mu-2}
\end{gathered}
$$




$$
\begin{aligned}
& \left\{\left(k+\mu \max \left\{b_{1},\left(b_{2}+\frac{c_{4}}{2 m_{1}}\right),\left(\frac{c_{4}}{2}+b_{3}\right)\right\}\right)\right. \\
& +\left(2 k-\mu \min \left\{\left(a_{1}-c_{2}\right),\left(a_{2}-c_{1}\right),\left(a_{3}-\frac{c_{3}}{m}\right)\right\}\right. \\
& +\mu \max \left\{\sigma_{1}^{2}+\frac{\sigma_{2}^{2}+\sigma_{5}^{2}}{n^{2}}, \sigma_{3}^{2}, \sigma_{4}^{2}+\frac{\sigma_{2}^{2}+\sigma_{5}^{2}}{m^{2}}\right\}+\mu \\
& \left.\cdot \max \left\{b_{1},\left(b_{2}+\frac{c_{4}}{2 m_{1}}\right),\left(\frac{c_{4}}{2}+b_{3}\right)\right\}\right) W+(k-\mu \\
& \cdot \min \left\{\left(a_{1}-c_{2}\right),\left(a_{2}-c_{1}\right),\left(a_{3}-\frac{c_{3}}{m}\right)\right\} \\
& \left.\left.+\frac{\mu(1+\mu)}{2}\left\{\sigma_{1}^{2}+\frac{\sigma_{2}^{2}+\sigma_{5}^{2}}{n^{2}}, \sigma_{3}^{2}, \sigma_{4}^{2}+\frac{\sigma_{2}^{2}+\sigma_{5}^{2}}{m^{2}}\right\}\right) W^{2}\right\} .
\end{aligned}
$$

Obviously, we can find a positive constant $K$ which satisfies

$$
L e^{k t}(1+W)^{\mu} \leqslant K e^{k t}
$$

Then

$$
\begin{aligned}
& d e^{k t}(1+W)^{\mu} \leqslant K e^{k t}-e^{k t} \mu W^{2}(1+W)^{\mu-1} \\
& \cdot\left(\sigma_{1} y_{1} d B_{1}(t)+\sigma_{3} y_{2} d B_{3}(t)+\sigma_{4} z d B_{4}(t)\right. \\
& \left.+\frac{y_{1} z}{1+m y_{1}+n z+k y_{1} z}\left(\sigma_{2} d B_{2}(t)+\sigma_{5} d B_{5}(t)\right)\right)
\end{aligned}
$$

We can integrate above inequality and then take expectation

$$
\begin{aligned}
\mathbf{E}\left[e^{k t}(1+W)^{\mu}\right] & \leqslant(1+W(0))^{\mu}+\frac{K}{k} e^{k t} \\
& =(1+W(0))^{\mu}+M_{1} e^{k t}
\end{aligned}
$$

where $M_{1}=K / k$, so

$$
\begin{aligned}
\lim _{t \rightarrow+\infty} \sup \mathbf{E}\left[W(t)^{\mu}\right] & \leqslant \lim _{t \rightarrow+\infty} \sup \mathbf{E}\left[(1+W(t))^{\mu}\right] \\
& \leqslant M_{1} .
\end{aligned}
$$

Since $\left(y_{1}(t)+y_{2}(t)+z(t)\right)^{\mu} \leqslant 3^{\mu}\left(y_{1}(t)+y_{2}(t)+z(t)\right)^{\mu / 2}=$ $3^{\mu}\left|y_{1}(t), y_{2}(t), z(t)\right|$, where $\left(y_{1}(t), y_{2}(t), z(t)\right) \in R_{+}^{3}$, therefore

$$
\begin{gathered}
\lim _{t \rightarrow+\infty} \sup \mathbf{E}\left[\frac{1}{\left|y_{1}(t), y_{2}(t), z(t)\right|^{\mu}}\right] \\
\leqslant 3^{\mu} \lim _{t \rightarrow+\infty} \sup \mathbf{E}\left[W(t)^{\mu}\right] \leqslant 3^{\mu} M_{1} .
\end{gathered}
$$

From Theorem 3, we have

$$
\lim _{t \rightarrow+\infty} \sup \mathbf{E}\left[\left|y_{1}(t), y_{2}(t), z(t)\right|^{p}\right] \leqslant K(p)
$$

for any $\varepsilon>0$; let $\chi=(K(p) / \varepsilon)^{1 / p}$, using Chebyshev's inequality, we can obtain the conclusion.

\section{Extinction}

The extinction of system (3) will be investigated in this part.

Definition 5. For arbitrary initial data $\gamma \in C\left([-\tau, 0], R_{+}^{3}\right)$, if

$$
\begin{aligned}
& \lim _{t \rightarrow+\infty} \sup \frac{\ln y_{1}(t)}{t}<0, \\
& \lim _{t \rightarrow+\infty} \sup \frac{\ln y_{2}(t)}{t}<0, \\
& \lim _{t \rightarrow+\infty} \sup \frac{\ln z(t)}{t}<0
\end{aligned}
$$

hold, then system (3) is extinct.

Theorem 6. For arbitrary initial data $\gamma \in C\left([-\tau, 0], R_{+}^{3}\right)$, assume that

$$
\begin{array}{r}
a_{1}-\frac{\sigma_{1}^{2}}{2}<0, \\
a_{2}-\frac{\sigma_{3}^{2}}{2}<0 \\
a_{3}+\frac{d_{1}}{m}+\frac{d_{2}^{2}}{2}+\frac{1}{2 m_{1}}-\frac{\sigma_{4}^{2}}{2}<0
\end{array}
$$

holds; by the definition of extinction, system (3) is said to be extinct.

Proof. We establish three Lyapunov functions as follows:

$$
\begin{gathered}
V_{1}\left(y_{1}(t)\right)=\ln y_{1}(t), \\
V_{2}\left(y_{2}(t)\right)=\ln y_{2}(t), \\
V_{3}(z(t))=\ln z(t) .
\end{gathered}
$$

Making use of the generalized Itô's formula results in

$$
\begin{aligned}
& d V_{1}\left(y_{1}\right)=\frac{d y_{1}}{y_{1}}-\frac{\left(d y_{1}\right)^{2}}{2 y_{1}^{2}}=\left(a_{1}-b_{1} y_{1}-\frac{c_{2} y_{2}\left(t-\tau_{2}\right)}{1+y_{2}\left(t-\tau_{2}\right)}\right. \\
& -\frac{c_{3} z}{1+m y_{1}+n z+k y_{1} z} \\
& \left.-\frac{1}{2}\left(\sigma_{1}^{2}+\frac{\sigma_{2}^{2} z^{2}}{\left(1+m y_{1}+n z+k y_{1} z\right)^{2}}\right)\right) d t \\
& +\sigma_{1} d B_{1}(t)+\frac{\sigma_{2} z}{1+m y_{1}+n z+k y_{1} z} d B_{2}(t)
\end{aligned}
$$




$$
\begin{aligned}
& d V_{2}\left(y_{2}\right)=\frac{d y_{2}}{y_{2}}-\frac{\left(d y_{2}\right)^{2}}{2 y_{2}^{2}}=\left(a_{2}-b_{2} y_{2}-\frac{c_{1} y_{1}\left(t-\tau_{1}\right)}{1+y_{1}\left(t-\tau_{1}\right)}\right. \\
& \left.-\frac{c_{4} z}{1+m_{1} y_{2}^{2}}-\frac{\sigma_{3}^{2}}{2}\right) d t+\sigma_{3} d B_{3}(t), \\
& d V_{3}(z)=\frac{d z}{z}-\frac{(d z)^{2}}{2 z^{2}}=\left(a_{3}-b_{3} z+\frac{d_{2} y_{2}\left(t-\tau_{3}\right)}{1+m_{1} y_{2}^{2}\left(t-\tau_{3}\right)}\right. \\
& +\frac{d_{1} y_{1}\left(t-\tau_{3}\right)}{1+m y_{1}\left(t-\tau_{3}\right)+n z\left(t-\tau_{3}\right)+k y_{1}\left(t-\tau_{3}\right) z\left(t-\tau_{3}\right)} \\
& \left.-\frac{1}{2}\left(\sigma_{4}^{2}+\frac{\sigma_{5}^{2} y_{1}^{2}}{\left(1+m y_{1}+n z+k y_{1} z\right)^{2}}\right)\right) d t \\
& +\sigma_{4} d B_{4}(t)+\frac{\sigma_{5} y_{1}}{1+m y_{1}+n z+k y_{1} z} d B_{5}(t) .
\end{aligned}
$$

Hence, we can integrate them from 0 to $t$ :

$$
\begin{aligned}
& \ln y_{1}(t)=\ln y_{1}(0)+\int_{0}^{t}\left(a_{1}-b_{1} y_{1}(s)-\frac{c_{2} y_{2}\left(s-\tau_{2}\right)}{1+y_{2}\left(s-\tau_{2}\right)}\right. \\
& -\frac{c_{3} z(s)}{1+m y_{1}(s)+n z(s)+k y_{1}(s) z(s)}-\frac{1}{2}\left(\sigma_{1}^{2}\right. \\
& \left.\left.+\frac{\sigma_{2}^{2} z^{2}(s)}{\left(1+m y_{1}(s)+n z(s)+k y_{1}(s) z(s)\right)^{2}}\right)\right) d s \\
& +\int_{0}^{t} \sigma_{1} d B_{1}(s)+M_{1}(t), \\
& +\int_{0}^{t} \sigma_{4} d B_{4}(s)+M_{2}(t), \\
& +\frac{1}{2}\left(\sigma_{4}^{2}=\ln y_{2}(0)+\int_{0}^{t}\left(a_{2}-b_{2} y_{2}(s)-\frac{c_{1} y_{1}\left(s-\tau_{1}\right)}{1+y_{1}\left(s-\tau_{1}\right)}\right.\right. \\
& \left.+\frac{c_{4} z(s)}{1+m_{1} y_{2}^{2}(s)}-\frac{\sigma_{3}^{2}}{2}\right) d s+\int_{0}^{t} \sigma_{3} d B_{3}(s) \\
& \ln z(t)=\ln z(0)+\int_{0}^{t}\left(a_{3}-b_{3} z(s)+\frac{d_{2} y_{2}\left(s-\tau_{3}\right)}{1+m_{1} y_{2}^{2}\left(s-\tau_{3}\right)}\right. \\
& +d_{1} y_{1}\left(s-\tau_{3}\right)
\end{aligned}
$$

where $M_{1}(t)=\int_{0}^{t}\left(\sigma_{2} z(s) /\left(1+m y_{1}(s)+n z(s)+\right.\right.$ $\left.\left.k y_{1}(s) z(s)\right)\right) d B_{2}(s)$ and $M_{2}(t)=\int_{0}^{t}\left(\sigma_{5} y_{1}(s) /\left(1+m y_{1}(s)+\right.\right.$ $\left.\left.n z(s)+k y_{1}(s) z(t)\right)\right) d B_{5}(s)$ are martingales. And

$$
\begin{aligned}
& \left\langle M_{1}(t), M_{1}(t)\right\rangle \\
& \quad=\int_{0}^{t}\left(\frac{\sigma_{2} y(s)}{1+m y_{1}(s)+n y(s)+k y_{1}(s) z(s)}\right)^{2} d s, \\
& \left\langle M_{2}(t), M_{2}(t)\right\rangle \\
& =\int_{0}^{t}\left(\frac{\sigma_{5} y_{1}(s)}{1+m y_{1}(s)+n z(s)+k y_{1}(s) z(s)}\right)^{2} d s
\end{aligned}
$$

are the quadratic variations, respectively. Using the exponential martingale inequality, for arbitrary positive constants $N, \eta$, and $v$, we can obtain

$$
P\left\{\sup _{0 \leqslant t \leqslant N}\left[M(t)-\frac{1}{2}\langle M(t), M(t)\rangle\right]>\beta\right\}=e^{-\eta \nu} .
$$

Choosing $N=n, \eta=1$, and $v=2 \ln n$, we have that

$$
\begin{aligned}
& P\left\{\sup _{0 \leqslant t \leqslant n}\left[M_{1}(t)-\frac{1}{2}\left\langle M_{1}(t), M_{1}(t)\right\rangle\right]>2 \ln n\right\} \\
& \leqslant \frac{1}{n^{2}}, \\
& P\left\{\sup _{0 \leqslant t \leqslant n}\left[M_{2}(t)-\frac{1}{2}\left\langle M_{2}(t), M_{2}(t)\right\rangle\right]>2 \ln n\right\} \\
& \quad \leqslant \frac{1}{n^{2}} .
\end{aligned}
$$

Applying the Borel-Cantelli lemma, there exists a random integer $n_{0}=n_{0}(\omega)$ for almost all $\omega \in \Omega$ such that

$$
\begin{aligned}
& \sup _{0 \leqslant t \leqslant n}\left[M_{1}(t)-\frac{1}{2}\left\langle M_{1}(t), M_{1}(t)\right\rangle\right] \leqslant 2 \ln n, \\
& \sup _{0 \leqslant t \leqslant n}\left[M_{2}(t)-\frac{1}{2}\left\langle M_{2}(t), M_{2}(t)\right\rangle\right] \leqslant 2 \ln n,
\end{aligned}
$$

for $n \geqslant n_{0}$.

Thus

$$
\begin{aligned}
& M_{1}(t) \\
& \leqslant 2 \ln n \\
& \quad+\frac{1}{2} \int_{0}^{t}\left(\frac{\sigma_{2} z(s)}{1+m y_{1}(s)+n z(s)+k y_{1}(s) z(s)}\right)^{2} d s, \\
& M_{2}(t) \\
& \leqslant 2 \ln n \\
& \quad+\frac{1}{2} \int_{0}^{t}\left(\frac{\sigma_{5} y_{1}(s)}{1+m y_{1}(s)+n z(s)+k y_{1}(s) z(s)}\right)^{2} d s,
\end{aligned}
$$


for all $0 \leqslant t \leqslant n, n \geqslant n_{0}$ a.s. We substitute it into (36)

$$
\begin{aligned}
\ln y_{1}(t)-\ln y_{1}(0) \leqslant & \left(a_{1}-\frac{1}{2} \sigma_{1}^{2}\right) t+\int_{0}^{t} \sigma_{1} d B_{1}(s) \\
& +2 \ln n, \\
\ln y_{2}(t)-\ln y_{2}(0) \leqslant & \left(a_{2}-\frac{1}{2} \sigma_{3}^{2}\right) t+\int_{0}^{t} \sigma_{3} d B_{3}(s), \\
\ln z(t)-\ln z(0) \leqslant & \left(a_{3}+\frac{d_{1}}{m}+\frac{d_{2}^{2}}{2}+\frac{1}{2 m_{1}}-\frac{\sigma_{4}^{2}}{2}\right) t \\
& +\int_{0}^{t} \sigma_{4} d B_{4}(s)+2 \ln n .
\end{aligned}
$$

We divide $t$ on both sides of (42) and let $t \rightarrow+\infty$ :

$$
\begin{aligned}
& \lim _{t \rightarrow+\infty} \sup \frac{\ln y_{1}(t)}{t} \leqslant a_{1}-\frac{\sigma_{1}^{2}}{2}<0, \\
& \lim _{t \rightarrow+\infty} \sup \frac{\ln y_{2}(t)}{t} \leqslant a_{2}-\frac{\sigma_{3}^{2}}{2}<0, \\
& \lim _{t \rightarrow+\infty} \sup \frac{\ln z(t)}{t} \leqslant a_{3}+\frac{d_{1}}{m}+\frac{d_{2}^{2}}{2}+\frac{1}{2 m_{1}}-\frac{\sigma_{4}^{2}}{2}<0 .
\end{aligned}
$$

The desired assertion is derived.

\section{Global Asymptotic Stability}

Definition 7. Let $\left(y_{1}(t), y_{2}(t), z(t)\right)$ and $\left(y_{1}^{*}(t), y_{2}^{*}(t), z^{*}(t)\right)$ be two arbitrary solutions of system (3) with initial data $\gamma, \gamma^{*} \in$ $\left([-\tau, 0], R_{+}^{3}\right)$, respectively. If

$$
\begin{aligned}
& P\left\{\operatorname { l i m } _ { t \rightarrow + \infty } \mathbf { E } \left[\mid\left(y_{1}(t), y_{2}(t), z(t)\right)\right.\right. \\
& \left.\left.\quad-\left(y_{1}^{*}(t), y_{2}^{*}(t), z^{*}(t)\right) \mid\right]=0\right\}=1,
\end{aligned}
$$

we say that system (3) is globally asymptotically stable in expectation.

Lemma 8. For any initial data $\gamma \in C\left([-\tau, 0], R_{+}^{3}\right),\left(y_{1}(t)\right.$, $\left.y_{2}(t), z(t)\right)$ is a solution of system (3); then almost every sample track of $\left(y_{1}(t), y_{2}(t), z(t)\right)$ is uniformly continuous for $t \geqslant-\tau$.

Proof. Considering system (3), we have that

$$
\begin{aligned}
y_{1}(t)= & y_{1}(0)+\int_{0}^{t} f_{1}(s) d s+\int_{0}^{t} g_{1}(s) d B_{1}(s) \\
& +\int_{0}^{t} g_{2}(s) d B_{2}(s)
\end{aligned}
$$

where

$$
\begin{gathered}
f_{1}(s)=y_{1}(s)\left(a_{1}-b_{1} y_{1}(s)-\frac{c_{2} y_{2}\left(s-\tau_{2}\right)}{1+y_{2}\left(s-\tau_{2}\right)}\right. \\
\left.-\frac{c_{3} z(s)}{1+m y_{1}(s)+n z(s)+k y_{1}(s) z(s)}\right), \\
g_{1}(s)=\sigma_{1} y_{1}(s), \\
g_{2}(s)=\frac{\sigma_{2} y_{1}(s) z(s)}{1+m y_{1}(s)+n z(s)+k y_{1}(s) z(s)} .
\end{gathered}
$$

Letting $\vartheta>2$, we obtain that

$$
\begin{aligned}
& \mathbf{E}\left[\left|f_{1}(t)\right|^{\vartheta}\right]=\mathbf{E}\left[\mid y_{1}\left(a_{1}-b_{1} y_{1}-\frac{c_{2} y_{2}\left(t-\tau_{2}\right)}{1+y_{2}\left(t-\tau_{2}\right)}\right.\right. \\
& \left.\left.-\frac{c_{3} z}{1+m y_{1}+n z+k y_{1} z}\right)\left.\right|^{9}\right] \leqslant \frac{1}{2} \mathbf{E}\left[\left|y_{1}\right|^{29}\right]+\frac{1}{2} \\
& \cdot 4^{29-1}\left[\left|a_{1}\right|^{2 \vartheta}+\left|b_{1}\right|^{2 \vartheta} \mathbf{E}\left[\left|y_{1}\right|^{2 \vartheta}\right]+\left|c_{2}\right|^{29} \mid\right. \\
& \left.\mathbf{E}\left[\left|y_{2}\left(t-\tau_{2}\right)\right|^{2 \vartheta}\right]+\left|c_{3}\right|^{2 \vartheta} \mathbf{E}\left[|z|^{29}\right]\right] \leqslant \frac{1}{2} K_{1}(2 \vartheta) \\
& +\frac{1}{2} 4^{2 \vartheta-1}\left[\left|a_{1}\right|^{2 \vartheta}+\left|b_{1}\right|^{2 \vartheta} K_{1}(2 \vartheta)+\left|c_{2}\right|^{2 \vartheta} K_{2}(2 \vartheta)\right. \\
& \left.+\left|c_{3}\right|^{2 \vartheta} K_{3}(2 \vartheta)\right] \triangleq L_{1}(\vartheta) \\
& \mathbf{E}\left[\left|g_{1}(s)\right|^{\vartheta}\right]=\mathbf{E}\left[\left|\sigma_{1}\right|^{\vartheta}\left|y_{1}\right|^{\vartheta}\right]=\left|\sigma_{1}\right|^{\vartheta} \mathbf{E}\left[\left|y_{1}\right|^{\vartheta}\right] \\
& \leqslant \sigma_{1}^{p} K_{1}(\vartheta) \triangleq L_{2}(\vartheta) \text {, } \\
& \mathbf{E}\left[\left|g_{2}(s)\right|^{\vartheta}\right]=\mathbf{E}\left[\left|\frac{\sigma_{2} y_{1} z}{1+m y_{1}+n z+k y_{1} z}\right|^{\vartheta}\right] \\
& \leqslant \mathbf{E}\left[\left|\frac{\sigma_{2} y_{1}}{n}\right|^{\vartheta}\right] \leqslant\left(\frac{\sigma_{2}}{n}\right)^{\vartheta} K_{1}(\vartheta) \triangleq L_{3}(\vartheta) \text {. }
\end{aligned}
$$

For another, using the moment inequality of stochastic integral (see [16]), for $0 \leqslant t_{1}<t_{2}<+\infty$ and $\vartheta>2$, we have that

$$
\begin{aligned}
& \mathbf{E}\left[\left|\int_{t_{1}}^{t_{2}} g_{1}(s) d B_{1}(s)\right|^{\vartheta}\right] \\
& \leqslant\left[\frac{\vartheta(\vartheta-1)}{2}\right]^{\vartheta / 2}\left(t_{2}-t_{1}\right)^{(\vartheta-2) / 2} \int_{t_{1}}^{t_{2}} \mathbf{E}\left[\left|g_{1}(s)\right|^{\vartheta}\right] d s \\
& \leqslant\left[\frac{\vartheta(\vartheta-1)}{2}\right]^{\vartheta / 2}\left(t_{2}-t_{1}\right)^{\vartheta / 2} L_{2}(\vartheta),
\end{aligned}
$$




$$
\begin{aligned}
& \mathbf{E}\left[\left|\int_{t_{1}}^{t_{2}} g_{2}(s) d B_{2}(s)\right|^{\vartheta}\right] \\
& \leqslant\left[\frac{\vartheta(\vartheta-1)}{2}\right]^{\vartheta / 2}\left(t_{2}-t_{1}\right)^{(\vartheta-2) / 2} \int_{t_{1}}^{t_{2}} \mathbf{E}\left[\left|g_{2}(s)\right|^{9}\right] d s \\
& \leqslant\left[\frac{\vartheta(\vartheta-1)}{2}\right]^{9 / 2}\left(t_{2}-t_{1}\right)^{9 / 2} L_{3}(\vartheta) .
\end{aligned}
$$

Thus for $t_{2}-t_{1}<1$ and $1 / \vartheta+1 / q=1$, we can obtain

$$
\begin{aligned}
& \mathbf{E}\left|y_{1}\left(t_{2}\right)-y_{1}\left(t_{1}\right)\right|^{9}=\mathbf{E}\left[\mid \int_{t_{1}}^{t_{2}} f_{1}(s) d s\right. \\
& \left.+\int_{t_{1}}^{t_{2}} g_{1}(s) d B_{1}(s)+\left.\int_{t_{1}}^{t_{2}} g_{2}(s) d B_{2}(s)\right|^{9}\right] \\
& \leqslant 3^{9-1}\left\{\mathbf{E}\left[\left|\int_{t_{1}}^{t_{2}} f_{1}(s) d s\right|\right]^{\vartheta}\right. \\
& +\mathbf{E}\left[\left|\int_{t_{1}}^{t_{2}} g_{1}(s) d B_{1}(s)\right|\right]^{\vartheta} \\
& \left.+\mathbf{E}\left[\left|\int_{t_{1}}^{t_{2}} g_{1}(s) d B_{1}(s)\right|\right]^{9}\right\} \leqslant 3^{9-1}\{1 \\
& \left.+\left[\frac{\vartheta(\vartheta-1)}{2}\right]^{9 / 2}\right\} L_{4}(\vartheta)\left(t_{2}-t_{1}\right)^{9 / 2}
\end{aligned}
$$

where $L_{4}(\vartheta)=\max \left\{L_{1}(\vartheta), L_{2}(\vartheta)+L_{3}(\vartheta)\right\}$. From Lemma 2.3 of [13], we have that almost every sample path of $y_{1}(t)$ is uniformly continuous. In the same method, the uniform continuity of $y_{2}(t)$ and $z(t)$ can be obtained.

Lemma 9 (see [17]). $g(t) \geqslant 0$ is a uniformly continuous and integrable function defined on $[0,+\infty)$; then $\lim _{t \rightarrow \infty} g(t)=0$.

Theorem 10. If

$$
\begin{aligned}
A: & =-b_{1}+c_{1}+2 d_{1}+\frac{2 \sigma_{5}^{2}}{m}+\frac{(m n+k) c_{3}}{n^{2}}+\frac{(m n+k) \sigma_{2}^{2}}{n^{3}}<0, \\
B: & -b_{2}+c_{2}+\frac{3 d_{2}}{2} \\
& +\frac{2 c_{4} m_{1}\left(a_{2}+\sigma_{3}^{2}\right)\left(a_{3}+d_{1} / m+d_{2}^{2} / 2+1 / 2 m_{1}+\sigma_{4}^{2}+\sigma_{5}^{2} / m^{2}\right)}{b_{2} b_{3}} \\
& <0, \\
C & :=-b_{3}+2 c_{3}+2 c_{4}+\frac{2 \sigma_{2}^{2}}{n}+\frac{(m n+k) d_{1}}{m^{2}}+\frac{(m n+k) \sigma_{5}^{2}}{m^{3}}<0,
\end{aligned}
$$

then system (3) is globally asymptotically stable.
Proof. $\left(y_{1}(t), y_{2}(t), z(t)\right)$ and $\left(y_{1}^{*}(t), y_{2}^{*}(t), z^{*}(t)\right)$ are two arbitrary solutions of system (3) with initial data $\gamma, \gamma^{*} \in$ $\left([-\tau, 0], R_{+}^{3}\right)$, respectively. Define

$$
\begin{aligned}
V(t)= & \left|\ln y_{1}-\ln y_{1}^{*}\right|+\left|\ln y_{2}-\ln y_{2}^{*}\right| \\
& +\left|\ln z-\ln z^{*}\right|+c_{1} \int_{t-\tau_{1}}^{t}\left|y_{1}(s)-y_{1}^{*}(s)\right| d s \\
& +c_{2} \int_{t-\tau_{2}}^{t}\left|y_{2}(s)-y_{2}^{*}(s)\right| d s \\
& +2 d_{1} \int_{t-\tau_{3}}^{t}\left|y_{1}(s)-y_{1}^{*}(s)\right| d s \\
& +\frac{3 d_{2}}{2} \int_{t-\tau_{3}}^{t}\left|y_{2}(s)-y_{2}^{*}(s)\right| d s \\
& +\frac{(m n+k) d_{1}}{m^{2}} \int_{t-\tau_{3}}^{t}\left|z(s)-z^{*}(s)\right| d s .
\end{aligned}
$$

Calculating the right differential $d^{+} V(t)$, by Itô's formula

$$
\begin{gathered}
d^{+} V=\operatorname{sgn}\left(y_{1}-y_{1}^{*}\right)\left\{\left[\left(a_{1}-b_{1} y_{1}-\frac{c_{2} y_{2}\left(t-\tau_{2}\right)}{1+y_{2}\left(t-\tau_{2}\right)}\right.\right.\right. \\
\left.-\frac{c_{3} z}{1+m y_{1}+n z+k y_{1} z}-\frac{\sigma_{2}^{2} z^{2}}{2\left(1+m y_{1}+n z+k y_{1} z\right)^{2}}\right) d t \\
\left.+\sigma_{1} d B_{1}(t)+\frac{\sigma_{2} z}{1+m y_{1}+n z+k y_{1} z} d B_{2}(t)\right]-\left[\left(a_{1}\right.\right. \\
-b_{1} y_{1}^{*}-\frac{c_{2} y_{2}^{*}\left(t-\tau_{2}\right)}{1+y_{2}^{*}\left(t-\tau_{2}\right)}-\frac{c_{3} z^{*}}{1+m y_{1}^{*}+n z^{*}+k y_{1}^{*} z^{*}}
\end{gathered}
$$$$
\left.-\frac{\sigma_{2}^{2}\left(z^{*}\right)^{2}}{2\left(1+m y_{1}^{*}+n z^{*}+k y_{1}^{*} z^{*}\right)^{2}}\right) d t+\sigma_{1} d B_{1}(t)
$$$$
\left.\left.+\frac{\sigma_{2} z^{*}}{1+m y_{1}^{*}+n z^{*}+k y_{1}^{*} z^{*}} d B_{2}(t)\right]\right\}+\operatorname{sgn}\left(y_{2}-y_{2}^{*}\right)
$$$$
\left\{\left[\left(a_{2}-b_{2} y_{2}-\frac{c_{1} y_{1}\left(t-\tau_{1}\right)}{1+y_{1}\left(t-\tau_{1}\right)}-\frac{c_{4} y}{1+m_{1} y_{2}^{2}}\right) d t\right.\right.
$$$$
\left.+\sigma_{3} d B_{3}(t)\right]-\left[\left(a_{2}-b_{2} y_{2}^{*}-\frac{c_{1} y_{1}^{*}\left(t-\tau_{1}\right)}{1+y_{1}^{*}\left(t-\tau_{1}\right)}\right.\right.
$$$$
\left.\left.\left.-\frac{c_{4} z^{*}}{1+m_{1}\left(y_{2}^{*}\right)^{2}}\right) d t+\sigma_{3} d B_{3}(t)\right]\right\}+\operatorname{sgn}\left(z-z^{*}\right)
$$

$\left\{\left[\left(a_{3}-b_{3} z+\frac{d_{2} y_{2}\left(t-\tau_{3}\right)}{1+m_{1} y_{2}^{2}\left(t-\tau_{3}\right)}\right.\right.\right.$ 


$$
\begin{aligned}
& +\frac{d_{1} y_{1}\left(t-\tau_{3}\right)}{1+m y_{1}\left(t-\tau_{3}\right)+n z\left(t-\tau_{3}\right)+k y_{1}\left(t-\tau_{3}\right) z\left(t-\tau_{3}\right)} \\
& \left.-\frac{\sigma_{5}^{2} y_{1}^{2}}{2\left(1+m y_{1}+n z+k y_{1} z\right)^{2}}\right) d t+\sigma_{4} d B_{4}(t) \\
& \left.+\frac{\sigma_{5} y_{1}}{1+m y_{1}+n z+k y_{1} z} d B_{5}(t)\right]-\left[\left(a_{3}-b_{3} z^{*}\right.\right. \\
& +\frac{d_{2} y_{2}^{*}\left(t-\tau_{3}\right)}{1+m_{1} y_{2}^{* 2}\left(t-\tau_{3}\right)} \\
& +\frac{d_{1} y_{1}^{*}\left(t-\tau_{3}\right)}{1+m y_{1}^{*}\left(t-\tau_{3}\right)+n z^{*}\left(t-\tau_{3}\right)+k y_{1}^{*}\left(t-\tau_{3}\right) z^{*}\left(t-\tau_{3}\right)} \\
& \left.-\frac{\sigma_{5}^{2} y_{1}^{* 2}}{2\left(1+m y_{1}^{*}+n z^{*}+k y_{1}^{*} z^{*}\right)^{2}}\right) d t+\sigma_{4} d B_{4}(t) \\
& +\frac{(m n+k) d_{1}}{m^{2}}\left[\left|z-z^{*}\right|-\left|z\left(t-\tau_{3}\right)-z^{*}\left(t-\tau_{3}\right)\right|\right] \\
& \left.\left.+\frac{\sigma_{5} y_{1}^{*}}{1+m y_{1}^{*}+n z^{*}+k y_{1}^{*} z^{*}} d B_{5}(t)\right]\right\}+c_{1}\left[\left|y_{1}-y_{1}^{*}\right|\right. \\
& \left.-\left|y_{1}\left(t-\tau_{1}\right)-y_{1}^{*}\left(t-\tau_{1}\right)\right|\right]+c_{2}\left[\left|y_{2}-y_{2}^{*}\right|-\mid y_{2}\left(t-\tau_{2}\right)\right. \\
& 2 d_{2}\left[\left|y_{2}-y_{2}^{*}\right|-\left|y_{2}\left(t-\tau_{3}\right)-y_{2}^{*}\left(t-\tau_{3}\right)\right|\right]
\end{aligned}
$$

We can take integration from 0 to $t$ and then take expectation

$$
\begin{aligned}
\mathbf{E}[V(t)] & -\mathbf{E}[V(0)] \\
= & \mathbf{E}\left\{\int _ { 0 } ^ { t } \left[\operatorname { s g n } ( y _ { 1 } ( s ) - y _ { 1 } ^ { * } ( s ) ) \left(-b_{1}\left(y_{1}(s)-y_{1}^{*}(s)\right)\right.\right.\right. \\
& -c_{2}\left(\frac{y_{2}\left(s-\tau_{2}\right)}{1+y_{2}\left(s-\tau_{2}\right)}-\frac{y_{2}^{*}\left(s-\tau_{2}\right)}{1+y_{2}^{*}\left(s-\tau_{2}\right)}\right) \\
& -c_{3}\left(\frac{z(s)}{1+m y_{1}(s)+n z(s)+k y_{1}(s) z(s)}\right. \\
& \left.-\frac{z^{*}(s)}{1+m y_{1}^{*}(s)+n z^{*}(s)+k y_{1}^{*}(s) z^{*}(s)}\right) \\
& -\frac{\sigma_{2}^{2}}{2}\left(\frac{z^{2}(s)}{\left(1+m y_{1}(s)+n z(s)+k y_{1}(s) z(s)\right)^{2}}\right. \\
& \left.\left.-\frac{\left(z^{*}(s)\right)^{2}}{\left(1+m y_{1}^{*}(s)+n z^{*}(s)+k y_{1}^{*}(s) z^{*}(s)\right)^{2}}\right)\right) \\
& +\operatorname{sgn}\left(y_{2}(s)-y_{2}^{*}(s)\right)\left(\begin{array}{l}
-b_{2}\left(y_{2}(s)-y_{2}^{*}(s)\right) \\
(1)
\end{array}\right.
\end{aligned}
$$

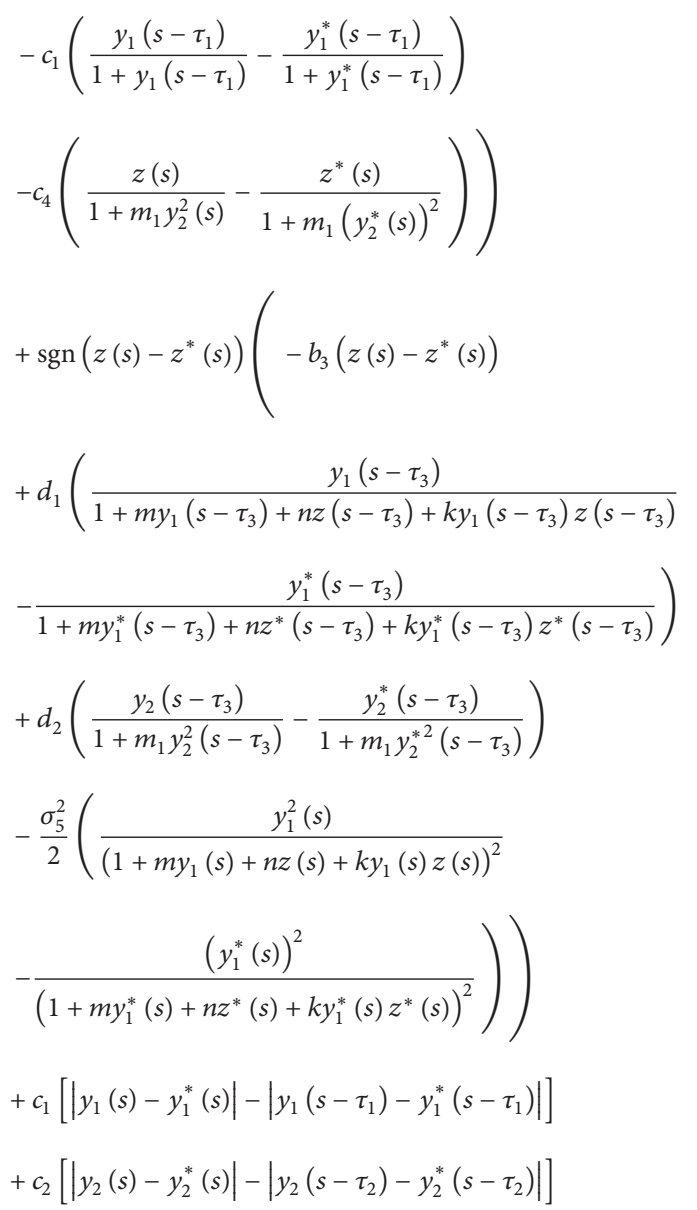$$
+2 d_{1}\left[\left|y_{1}(s)-y_{1}^{*}(s)\right|-\left|y_{1}\left(s-\tau_{3}\right)-y_{1}^{*}\left(s-\tau_{3}\right)\right|\right]
$$$$
+\frac{3 d_{2}}{2}\left[\left|y_{2}(s)-y_{2}^{*}(s)\right|-\left|y_{2}\left(s-\tau_{3}\right)-y_{2}^{*}\left(s-\tau_{3}\right)\right|\right]
$$$$
+\frac{(m n+k) d_{1}}{m^{2}}
$$$$
\left.\left.\cdot\left[\left|z(s)-z^{*}(s)\right|-\left|z\left(s-\tau_{3}\right)-z^{*}\left(s-\tau_{3}\right)\right|\right]\right] d s\right\} .
$$

Hence,

$$
\begin{aligned}
& \frac{d \mathbf{E}[V(t)]}{d t} \leqslant\left[-b_{1}+c_{1}+2 d_{1}+\frac{2 \sigma_{5}^{2}}{m}+\frac{(m n+k) c_{3}}{n^{2}}\right. \\
& \left.+\frac{(m n+k) \sigma_{2}^{2}}{n^{3}}\right] \mathbf{E}\left[\left|y_{1}-y_{1}^{*}\right|\right]+\left[-b_{2}+c_{2}+\frac{3 d_{2}}{2}\right. \\
& +c_{4} m_{1}\left(\left[\mathbf{E}|z|^{3}\right]^{1 / 3}\left[\mathbf{E}\left|y_{2}\right|^{3}\right]^{1 / 3}\right. \\
& \left.\left.+\left[\mathbf{E}|z|^{3}\right]^{1 / 3}\left[\mathbf{E}\left|y_{2}^{*}\right|^{3}\right]^{1 / 3}\right)\left[\mathbf{E}\left|y_{2}-y_{2}^{*}\right|\right]\right]+\left[-b_{3}\right.
\end{aligned}
$$




$$
\begin{array}{lcl}
\left.+2 c_{3}+2 c_{4}+\frac{2 \sigma_{2}^{2}}{n}+\frac{(m n+k) d_{1}}{m^{2}}+\frac{(m n+k) \sigma_{5}^{2}}{m^{3}}\right] & {\left[\mathbf{E}\left|y_{2}(t)\right|^{3}\right]^{1 / 3} \leqslant \frac{a_{2}+\sigma_{3}^{2}}{b_{2}},} \\
\cdot \mathbf{E}\left[\left|z-z^{*}\right|\right] \cdot & {\left[\mathbf{E}|z(t)|^{3}\right]^{1 / 3}} \\
\text { By Lemma 2, we have } & \leqslant \frac{a_{3}+d_{1} / m+d_{2}^{2} / 2+1 / 2 m_{1}+\sigma_{4}^{2}+\sigma_{5}^{2} / m^{2}}{b_{3}} .
\end{array}
$$

$$
\left[\mathbf{E}\left|y_{1}(t)\right|^{3}\right]^{1 / 3} \leqslant \frac{a_{1}+\sigma_{1}^{2}+\sigma_{2}^{2} / n^{2}}{b_{1}}
$$

Thus

$$
\begin{aligned}
\frac{d \mathbf{E}[V(t)]}{d t} \leqslant & \left(-b_{1}+c_{1}+2 d_{1}+\frac{2 \sigma_{5}^{2}}{m}+\frac{(m n+k) c_{3}}{n^{2}}+\frac{(m n+k) \sigma_{2}^{2}}{n^{3}}\right) \mathbf{E}\left[\left|y_{1}(t)-y_{1}^{*}(t)\right|\right] \\
& +\left(-b_{2}+c_{2}+\frac{3 d_{2}}{2}+\frac{2 c_{4} m_{1}\left(a_{2}+\sigma_{3}^{2}\right)\left(a_{3}+\sigma_{4}^{2}+d_{1} / m+d_{2}^{2} / 2+1 / 2 m_{1}+\sigma_{5}^{2} / m^{2}\right)}{b_{2} b_{3}}\right) \mathbf{E}\left[\left|y_{2}(t)-y_{2}^{*}(t)\right|\right] \\
& +\left(-b_{3}+2 c_{3}+2 c_{4}+\frac{2 \sigma_{2}^{2}}{n}+\frac{(m n+k) d}{m^{2}}+\frac{(m n+k) \sigma_{5}^{2}}{m^{3}}\right) \mathbf{E}\left[\left|z(t)-z^{*}(t)\right|\right] \\
= & A \mathbf{E}\left[\left|y_{1}(t)-y_{1}^{*}(t)\right|\right]+B \mathbf{E}\left[\left|y_{2}(t)-y_{2}^{*}(t)\right|\right]+C \mathbf{E}\left[\left|z(t)-z^{*}(t)\right|\right] .
\end{aligned}
$$

Integrating both sides of (57) yields that

$$
\begin{aligned}
V(t)-V(0) \leqslant & A \int_{0}^{t} \mathbf{E}\left[\left|y_{1}(s)-y_{1}^{*}(s)\right|\right] d s \\
& +B \int_{0}^{t} \mathbf{E}\left[\left|y_{2}(s)-y_{2}^{*}(s)\right|\right] d s \\
& +C \int_{0}^{t} \mathbf{E}\left[\left|z(s)-z^{*}(s)\right|\right] d s .
\end{aligned}
$$

Therefore

$$
\begin{aligned}
& V(t)-\left(A \int_{0}^{t} \mathbf{E}\left[\left|y_{1}(s)-y_{1}^{*}(s)\right|\right] d s\right. \\
& +B \int_{0}^{t} \mathbf{E}\left[\left|y_{2}(s)-y_{2}^{*}(s)\right|\right] d s \\
& \left.+C \int_{0}^{t} \mathbf{E}\left[\left|z(s)-z^{*}(s)\right|\right] d s\right) \leqslant V(0)<+\infty
\end{aligned}
$$

Then we have

$$
\begin{aligned}
& \mathbf{E}\left[\left|\left(y_{1}(t), y_{2}(t), z(t)\right)-\left(y_{1}^{*}(t), y_{2}^{*}(t), z^{*}(t)\right)\right|\right] \\
& \quad \leqslant \mathbf{E}\left[\left|y_{1}(t)-y_{1}^{*}(t)\right|+\left|y_{2}(t)-y_{2}^{*}(t)\right|\right. \\
& \left.\quad+\left|z(t)-z^{*}(t)\right|\right] \in L^{1}[0,+\infty) .
\end{aligned}
$$

Thus it follows from Lemma 9 that

$$
\begin{aligned}
& \lim _{t \rightarrow \infty}\left|y_{1}(t)-y_{1}^{*}(t)\right|=0, \\
& \lim _{t \rightarrow \infty}\left|y_{2}(t)-y_{2}^{*}(t)\right|=0, \\
& \lim _{t \rightarrow \infty}\left|z(t)-z^{*}(t)\right|=0 .
\end{aligned}
$$

7. Numerical Simulation

We give some illustrative examples to evidence our results in this part. Using the Milstein method mentioned in ([18]), we get the discretization equations of system (3):

$$
\begin{gathered}
y_{1, i+1}=y_{1, i}+y_{1, i}\left(a_{1}-b_{1} y_{1, i}-\frac{c_{2} y_{2,\left(i-s_{2}\right)}}{1+y_{2,\left(i-s_{2}\right)}}\right) \Delta t+\sigma_{1} y_{1, i} \sqrt{\Delta t} \eta_{1, i} \\
\left.-\frac{c_{3} z_{i}}{1+m y_{1, i}+n z_{i}+k y_{1, i} z_{i}}\right) \\
+\frac{\sigma_{2} y_{1, i} z_{i}}{1+m y_{1, i}+n z_{i}+k y_{1, i} z_{i}} \sqrt{\Delta t} \eta_{2, i}+\frac{\sigma_{1}^{2}}{2} y_{1, i}\left(\eta_{1, i}^{2}\right. \\
-1) \Delta t+\frac{\sigma_{2}^{2} y_{1, i} z_{i}^{2}\left(1+n z_{i}\right)}{2\left(1+m y_{1, i}+n z_{i}+k y_{1, i} z_{i}\right)^{3}}\left(\eta_{2, i}^{2}-1\right)
\end{gathered}
$$

$\cdot \Delta t$ 


$$
\begin{aligned}
& y_{2, i+1}=y_{2, i}+y_{2, i}\left(a_{2}-b_{2} y_{2, i}-\frac{c_{1} y_{1,\left(i-s_{1}\right)}}{1+y_{1,\left(i-s_{1}\right)}}\right. \\
& \left.-\frac{c_{4} z_{i}}{1+m_{1} y_{2, i}^{2}}\right) \Delta t+\sigma_{3} y_{2, i} \sqrt{\Delta t} \eta_{3, i}+\frac{\sigma_{3}^{2}}{2} y_{2, i}\left(\eta_{3, i}^{2}\right. \\
& \text { - 1) } \Delta t \text {, } \\
& z_{i+1}=z_{i}+z_{i}\left(a_{3}-b_{3} z_{i}\right. \\
& +\frac{d_{1} y_{1,\left(i-s_{3}\right)}}{1+m y_{1,\left(i-s_{3}\right)}+n z_{i-s_{3}}+k y_{1,\left(i-s_{3}\right)} z_{i-s_{3}}} \\
& \left.+\frac{d_{2} y_{2,\left(i-s_{3}\right)}}{1+m_{1} y_{2,\left(i-s_{3}\right)}^{2}}\right) \Delta t+\sigma_{4} z_{i} \sqrt{\Delta t} \eta_{4, i} \\
& +\frac{\sigma_{5} y_{1, i} z_{i}}{1+m y_{1, i}+n z_{i}+k y_{1, i} z_{i}} \sqrt{\Delta t} \eta_{5, i}+\frac{\sigma_{4}^{2}}{2} z_{i}\left(\eta_{4, i}^{2}\right.
\end{aligned}
$$

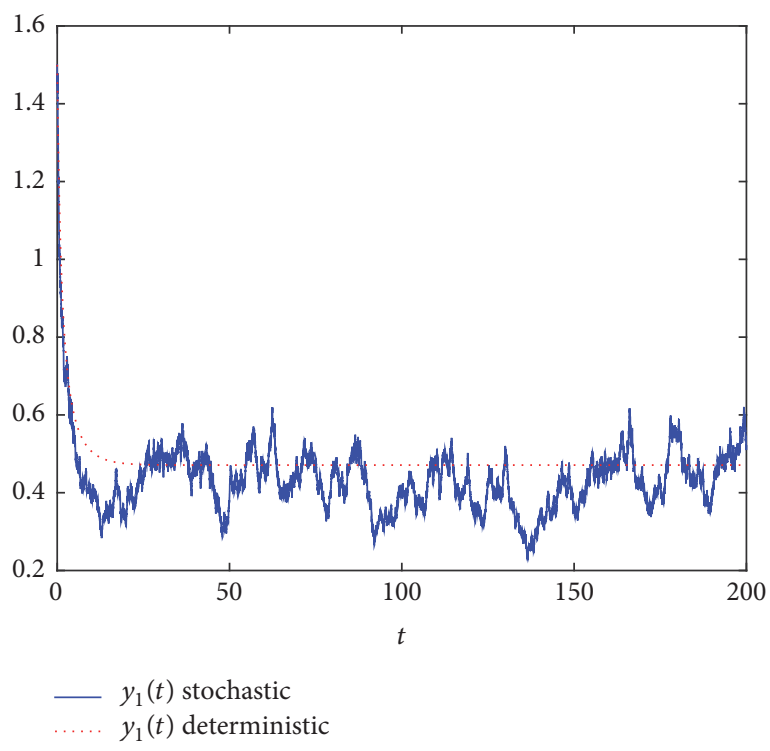$$
\text { - 1) } \Delta t+\frac{\sigma_{5}^{2} y_{1, i}^{2} z_{i}\left(1+m_{1} y_{1, i}\right)}{2\left(1+m y_{1, i}+n z_{i}+k y_{1, i} z_{i}\right)^{3}}\left(\eta_{5, i}^{2}-1\right)
$$$$
\cdot \Delta t
$$

where $\eta_{1, i}, \eta_{2, i}, \eta_{3, i}, \eta_{4, i}$, and $\eta_{5, i}$ are independent Gaussian random variables which follow $N(0,1)$. Let step size $\Delta t=$ 0.01 and steps $=20000$. We choose

$$
\begin{aligned}
& a_{1}=0.4 ; \\
& b_{1}=0.5 ; \\
& c_{2}=0.2 ; \\
& c_{3}=0.2 ; \\
& m=1 ; \\
& n=0.8 ; \\
& k=0.1 ; \\
& a_{2}=0.5 ; \\
& b_{2}=0.55 ; \\
& c_{1}=0.1 ; \\
& c_{4}=0.1 ; \\
& m_{1}=1 ; \\
& a_{3}=0.6 ; \\
& b_{3}=0.7 ; \\
& d_{1}=0.02 ; \\
& d_{2}=0.01 ;
\end{aligned}
$$

FIGURE 1: Solution of model $(62)$ for $\gamma_{1}(\theta)=(1.5,1.3,1)$. Blue: $\sigma_{i}=$ $0.1(i=1,2,3,4,5)$. Red: $\sigma_{i}=0(i=1,2,3,4,5)$.

$$
\begin{aligned}
& \tau_{1}=0.1 ; \\
& \tau_{2}=0.2 ; \\
& \tau_{3}=0.3 .
\end{aligned}
$$

We assume that the parameters are the same above in the following discussion. Let the initial data be

$$
\gamma_{1}(\theta)=(1.5,1.3,1), \quad-\tau \leqslant \theta \leqslant 0,
$$

where $\tau=\max \left\{\tau_{1}, \tau_{2}, \tau_{3}\right\}$. We choose $\sigma_{1}=\sigma_{2}=\sigma_{3}=$ $\sigma_{4}=\sigma_{5}=0.1$; from Theorem 3 we know that system (62) is stochastically ultimately bounded (see Figures 1-3: blue curves). To understand the influence of white noise, we choose $\sigma_{1}=\sigma_{2}=\sigma_{3}=\sigma_{4}=\sigma_{5}=0$; we obtain deterministic system of system (62) and it is also ultimately bounded (see Figures 1-3: red curves).

For stochastic permanence, we also choose $\sigma_{1}=\sigma_{2}=$ $\sigma_{3}=\sigma_{4}=\sigma_{5}=0.1$; by calculating we obtain that

$$
\begin{aligned}
\min & \left\{a_{1}-c_{2}, a_{2}-c_{1}, a_{3}-\frac{c_{3}}{m}\right\} \\
& -\frac{1}{2} \max \left\{\sigma_{1}^{2}+\frac{\sigma_{2}^{2}+\sigma_{5}^{2}}{n^{2}}, \sigma_{3}^{2}, \sigma_{4}^{2}+\frac{\sigma_{2}^{2}+\sigma_{5}^{2}}{m^{2}}\right\} \\
= & \frac{287}{1600},
\end{aligned}
$$

which satisfies the condition (16) (see Figure 4).

In the following we choose larger noises $\sigma_{1}=0.95, \sigma_{2}=$ $0.1, \sigma_{3}=1.05, \sigma_{4}=1.5$, and $\sigma_{5}=0.1$, which satisfy the condition of Theorem 6 ; then system (62) will go to extinction (see Figure 5). 


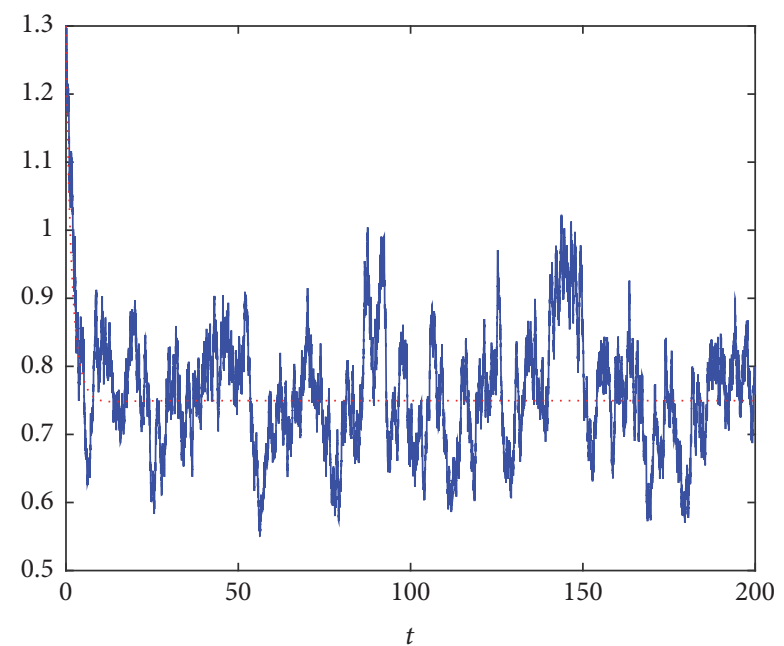

$\begin{array}{ll}- & y_{2}(t) \text { stochastic } \\ \ldots \ldots & y_{2}(t) \text { deterministic }\end{array}$

FIGURE 2: Solution of model (62) for $\gamma_{1}(\theta)=(1.5,1.3,1)$. Blue: $\sigma_{i}=$ $0.1(i=1,2,3,4,5)$. Red: $\sigma_{i}=0(i=1,2,3,4,5)$.

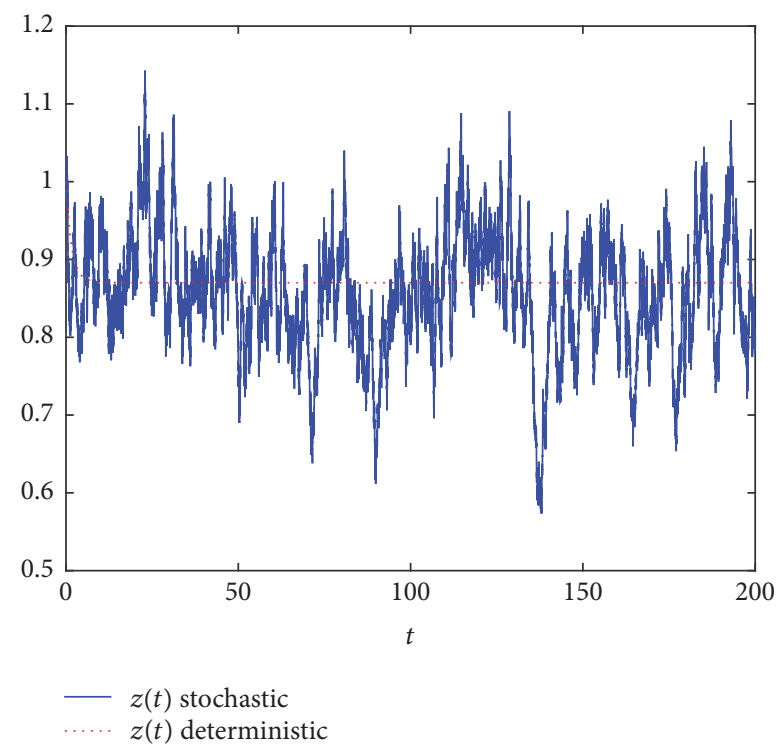

FIGURE 3: Solution of model $(62)$ for $\gamma_{1}(\theta)=(1.5,1.3,1)$. Blue: $\sigma_{i}=$ $0.1(i=1,2,3,4,5)$. Red: $\sigma_{i}=0(i=1,2,3,4,5)$.

In the end, we discuss the global asymptotic stability. Let

$$
\gamma_{1}^{*}=(0.7,0.8,0.6), \quad-\tau \leqslant \theta \leqslant 0 ;
$$

we also choose $\sigma_{1}=\sigma_{2}=\sigma_{3}=\sigma_{4}=\sigma_{5}=0.1$; by calculating we have that

$$
\begin{aligned}
A & :=-b_{1}+c_{1}+2 d_{1}+\frac{2 \sigma_{5}^{2}}{m}+\frac{(m n+k) c_{3}}{n^{2}}+\frac{(m n+k) \sigma_{2}^{2}}{n^{3}} \\
& =-\frac{527}{12800},
\end{aligned}
$$
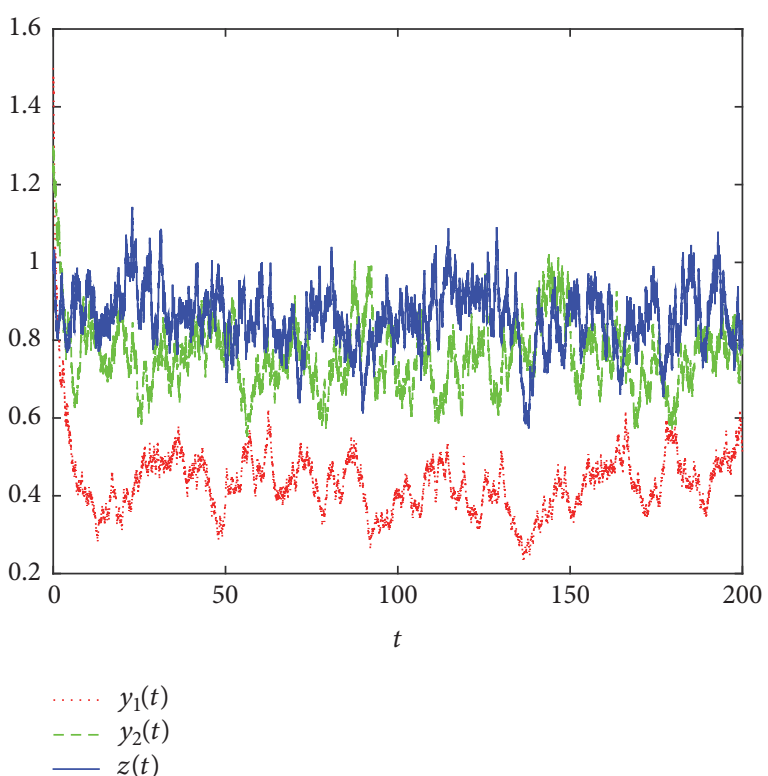

Figure 4: Solution of model $(62)$ for $\gamma_{1}(\theta)=(1.5,1.3,1)$ and $\sigma_{i}=$ $0.1(i=1,2,3,4,5)$.

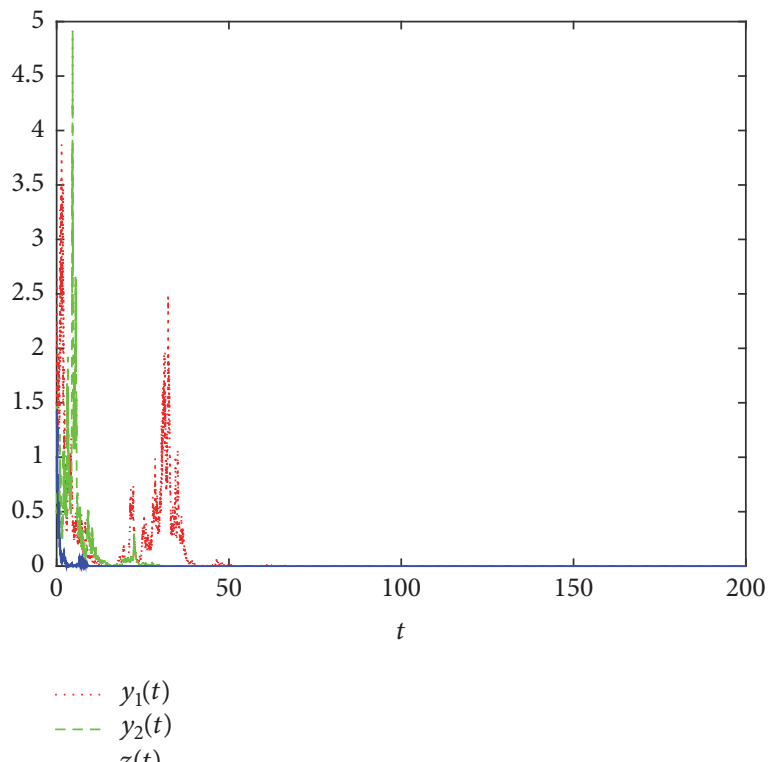

FIGURE 5: Solution of model $(62)$ for $\gamma_{1}(\theta)=(1.5,1.3,1)$ and $\sigma_{1}=$ $0.95, \sigma_{2}=0.1, \sigma_{3}=1.05, \sigma_{4}=1.5$, and $\sigma_{5}=0.1$.

$$
\begin{aligned}
B & :=-b_{2}+c_{2}+\frac{3 d_{2}}{2} \\
& +\frac{2 c_{4} m_{1}\left(a_{2}+\sigma_{3}^{2}\right)\left(a_{3}+d_{1} / m+d_{2}^{2} / 2+1 / 2 m_{1}+\sigma_{4}^{2}+\sigma_{5}^{2} / m^{2}\right)}{b_{2} b_{3}} \\
& =-\frac{174}{5279}, \\
C & :=-b_{3}+2 c_{3}+2 c_{4}+\frac{2 \sigma_{2}^{2}}{n}+\frac{(m n+k) d_{1}}{m^{2}}+\frac{(m n+k) \sigma_{5}^{2}}{m^{3}} \\
& =-\frac{6}{125},
\end{aligned}
$$



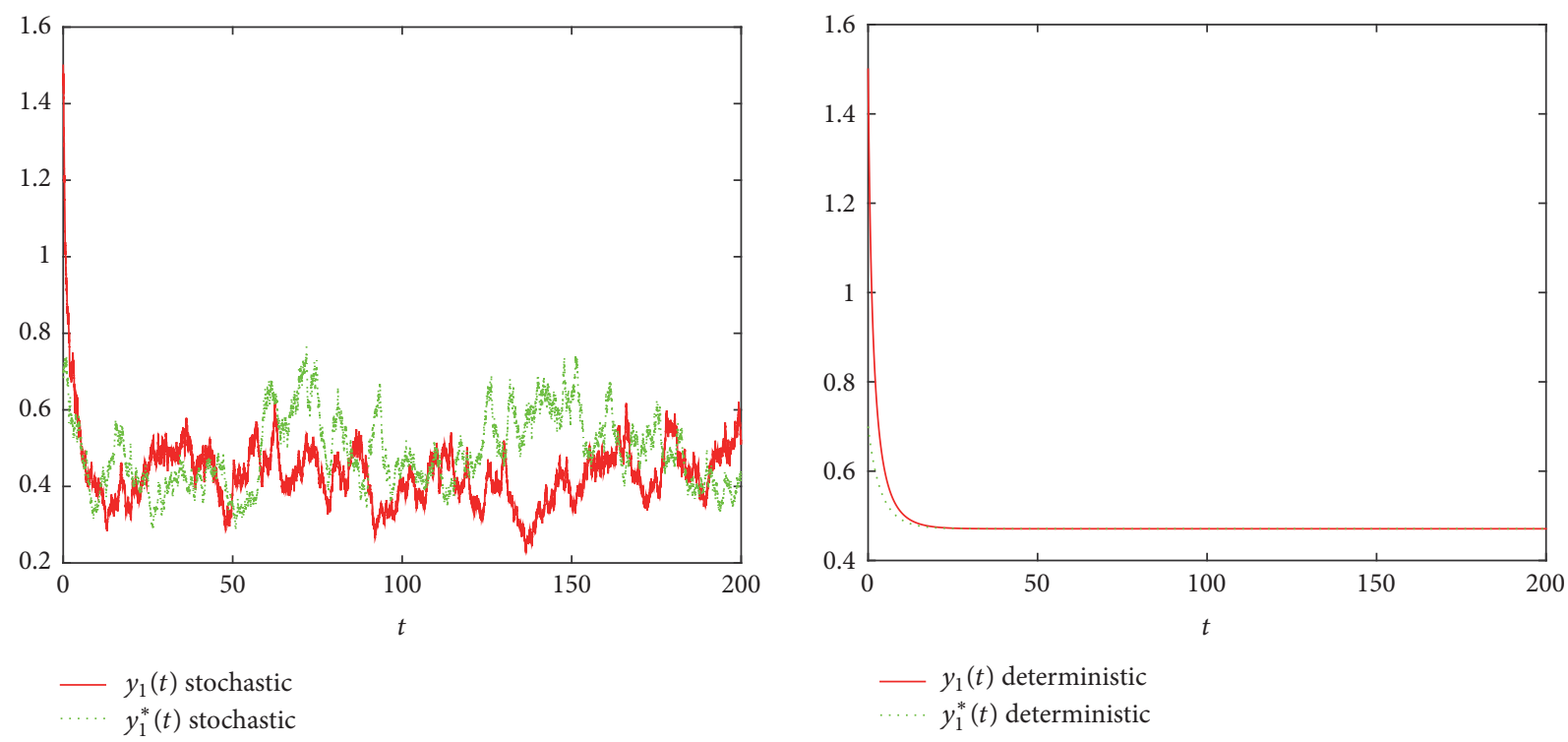

FIGURE 6: The path curve of $y_{1}(t)$ and $y_{1}^{*}(t)$. Red: solution of model $(62)$ for $\gamma_{1}(\theta)=(1.5,1.3,1)$. Green: solution of model $(62)$ for $\gamma_{1}^{*}=$ $(0.7,0.8,0.6)$.
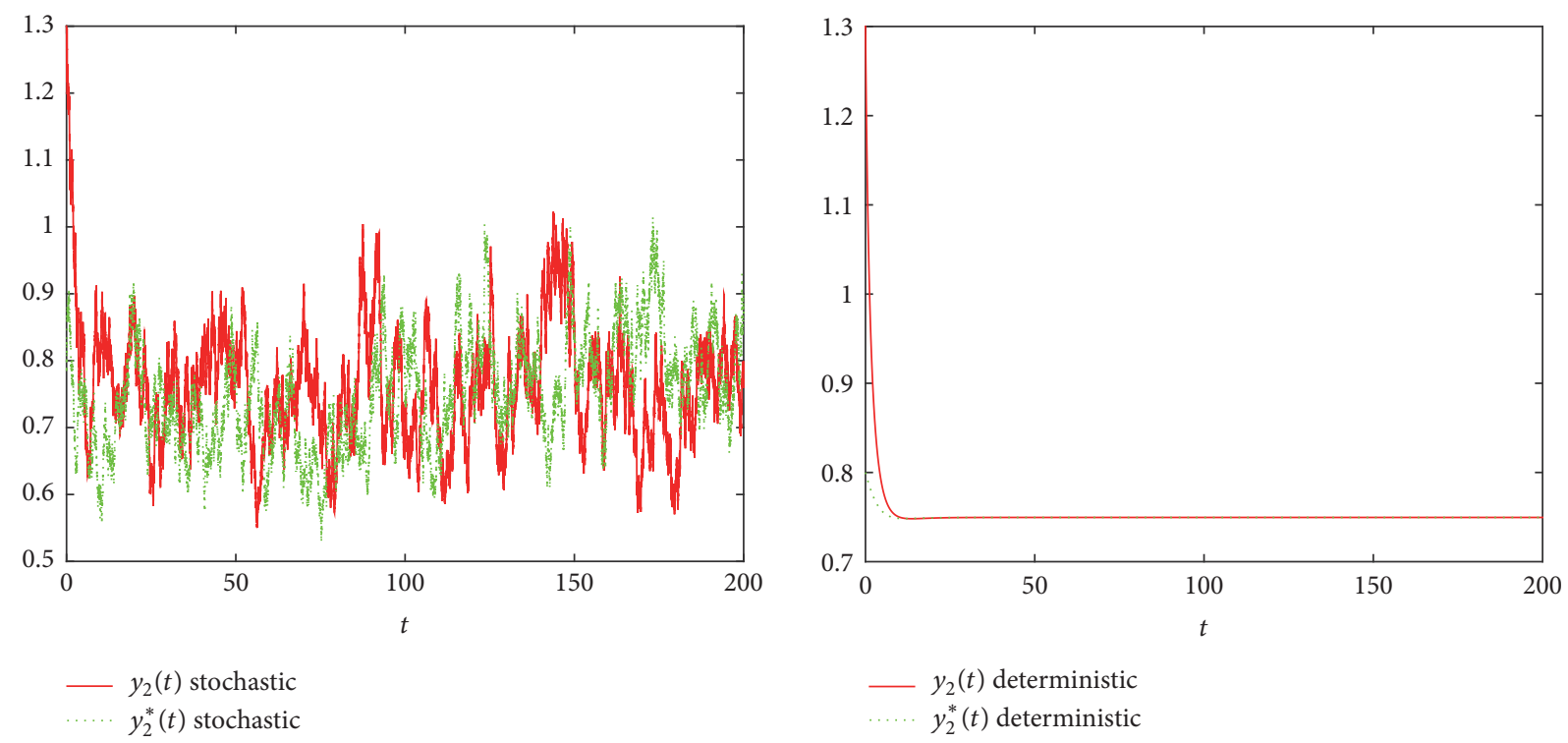

Figure 7: The path curve of $y_{2}(t)$ and $y_{2}^{*}(t)$. Red: solution of model $(62)$ for $\gamma_{1}(\theta)=(1.5,1.3,1)$. Green: solution of model $(62)$ for $\gamma_{1}^{*}=$ $(0.7,0.8,0.6)$.

which satisfy the condition of Theorem 10; then system (62) is globally asymptotically stable. By the same way, we can obtain the fact that the deterministic system of (3) is also globally stable (see Figures 6-8).

\section{Conclusions and Discussion}

In this work, stochastic delayed one-predator and twocompeting-prey systems with two different kinds of functional responses have been studied. Globally positive solution, stochastically ultimate boundedness, and the stochastic permanence and extinction for system (3) are investigated. Moreover, sufficient criteria for the global asymptotic stability of the system are established. In the end, some numerical examples are provided to explain our results. Through the study of the dynamic behavior of system (3), by comparing Theorems 4 and 6, we can obtain that if the environment noise is small, the stochastic system can maintain permanent while the system can be extinct under sufficiently large environmental noise (see Figures 4 and 5). Therefore, from Theorem 10, we can choose the appropriate parameters in a suitable environment noise intensity to make system (3) asymptotically stable. 

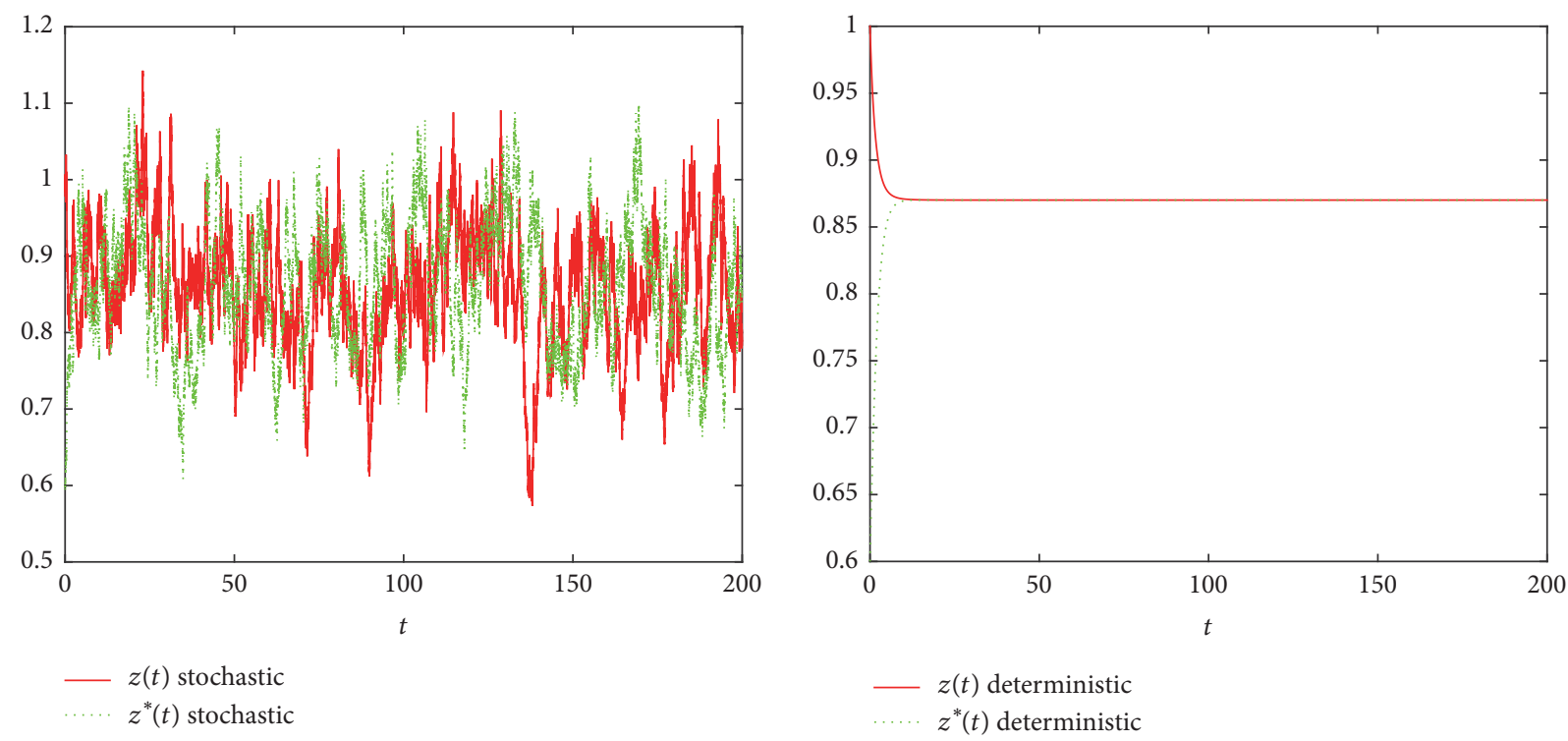

Figure 8: The path curve of $z(t)$ and $z^{*}(t)$. Red: solution of model $(62)$ for $\gamma_{1}(\theta)=(1.5,1.3,1)$. Green: solution of model $(62)$ for $\gamma_{1}^{*}=$ $(0.7,0.8,0.6)$.

Recently, predator-prey models have been investigated extensively for their theoretical and practical significance. In the current literatures, most of this work is restricted to two-dimensional predator-prey system; few has been done on predator-prey system with interspecific competition in preys. In system (3), we study a three-dimensional hybrid system where the predator can capture two kinds of preys with different functional responses. In fact, the population models are often subjected to the influence of environmental noises inevitably. In most of predator-prey models, there is only a white noise which affects intrinsic rate of increase of predator or prey. In system (3), we consider the white noise not only has effect on the intrinsic rate of population growth but also on capture rate of predator and conversion rate of the predator population. Three time delays are introduced to make the model closer to the reality. It is interesting to point out that system (3) contains two kinds of different mathematical models; if we remove the predator, system (3) is reduced to a competitive model in [13]; if we remove any one of the two preys system (3) is reduced to a simple predator-prey system. Some meaningful questions deserve further investigation. One may investigate the stationary distribution of system (3). Moreover, it is worth considering the corresponding nonautonomous system of system (3). One may discuss dynamics behaviors contained predators with a mutual cooperation in other ecosystems.

\section{Competing Interests}

The authors declare that they have no competing interests.

\section{Acknowledgments}

The authors thank the National Natural Science Foundation of China (nos. 61563033 and 11563005), Natural Science
Foundation of Jiangxi Province (nos. 20122BAB201002 and 20151BAB212011), and Innovation Fund Designated for Graduate Students of Nanchang University (no. cx2015089) for their financial support.

\section{References}

[1] A. A. Berryman, "The origins and evolution of predator-prey theory," Ecology, vol. 73, no. 5, pp. 1530-1535, 1992.

[2] X. Shi, X. Zhou, and X. Song, "Analysis of a stage-structured predator-prey model with Crowley-Martin function," Journal of Applied Mathematics and Computing, vol. 36, no. 1-2, pp. 459472, 2011.

[3] Z. She and H. Li, "Dynamics of a density-dependent stagestructured predator-prey system with Beddington-DeAngelis functional response," Journal of Mathematical Analysis and Applications, vol. 406, no. 1, pp. 188-202, 2013.

[4] S. Li, Z. Xiong, and X. Wang, "The study of a predator-prey system with group defense and impulsive control strategy," Applied Mathematical Modelling. Simulation and Computation for Engineering and Environmental Systems, vol. 34, no. 9, pp. 2546-2561, 2010.

[5] J. Huang, S. Ruan, and J. Song, "Bifurcations in a predatorprey system of Leslie type with generalized Holling type III functional response," Journal of Differential Equations, vol. 257, no. 6, pp. 1721-1752, 2014.

[6] C. Ji, D. Jiang, and N. Shi, "Analysis of a predator-prey model with modified Leslie-Gower and Holling-type II schemes with stochastic perturbation," Journal of Mathematical Analysis and Applications, vol. 359, no. 2, pp. 482-498, 2009.

[7] Q. Zhang and D. Jiang, "The coexistence of a stochastic LotkaVolterra model with two predators competing for one prey," Applied Mathematics and Computation, vol. 269, Article ID 21456, pp. 288-300, 2015.

[8] J. P. Tripathi, S. Tyagi, and S. Abbas, "Global analysis of a delayed density dependent predator-prey model with Crowley-Martin 
functional response," Communications in Nonlinear Science and Numerical Simulation, vol. 30, no. 1-3, pp. 45-69, 2016.

[9] M. Liu, C. Bai, M. Deng, and B. Du, "Analysis of stochastic two-prey one-predator model with Levy jumps," Physica A. Statistical Mechanics and its Applications, vol. 445, pp. 176-188, 2016.

[10] H. I. Freedman and P. Waltman, "Mathematical analysis of some three-species food-chain models," Mathematical Biosciences, vol. 33, no. 3-4, pp. 257-276, 1977.

[11] M. Liu and K. Wang, "Dynamics of a two-prey one-predator system in random environments," Journal of Nonlinear Science, vol. 23, no. 5, pp. 751-775, 2013.

[12] M. Ouyang and X. Li, "Permanence and asymptotical behavior of stochastic prey-predator system with Markovian switching," Applied Mathematics and Computation, vol. 266, pp. 539-559, 2015.

[13] R. Tan, H. Xiang, Y. Chen, and Z. Liu, "Dynamics behaviors of a delayed competitive system in a random environment," International Journal of Biomathematics, no. 5, pp. 181-199, 2015.

[14] Q. Liu, Y. Liu, and X. Pan, "Global stability of a stochastic predator-prey system with infinite delays," Applied Mathematics and Computation, vol. 235, pp. 1-7, 2014.

[15] M. Liu and C. Bai, "Global asymptotic stability of a stochastic delayed predator-prey model with Beddington-DeAngelis functional response," Applied Mathematics and Computation, vol. 226, pp. 581-588, 2014.

[16] X. Mao, Stochastic Differential Equations and Applications, Horwood Publishing, Chichester, UK, 1997.

[17] I. Barbalat, "Systems d'equations differentielle d'oscillations nonlinearires," Revue Roumaine de Mathematiques Pures et Appliquees, vol. 4, pp. 267-270, 1959.

[18] D. J. Higham, "An algorithmic introduction to numerical simulation of stochastic differential equations," SIAM Review, vol. 43, no. 3, pp. 525-546, 2001. 


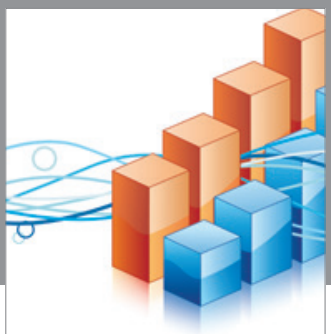

Advances in

Operations Research

vatem alat4

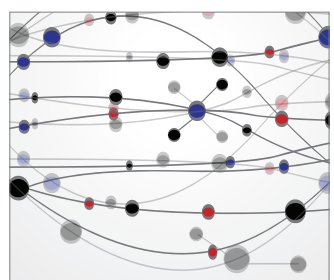

\section{The Scientific} World Journal
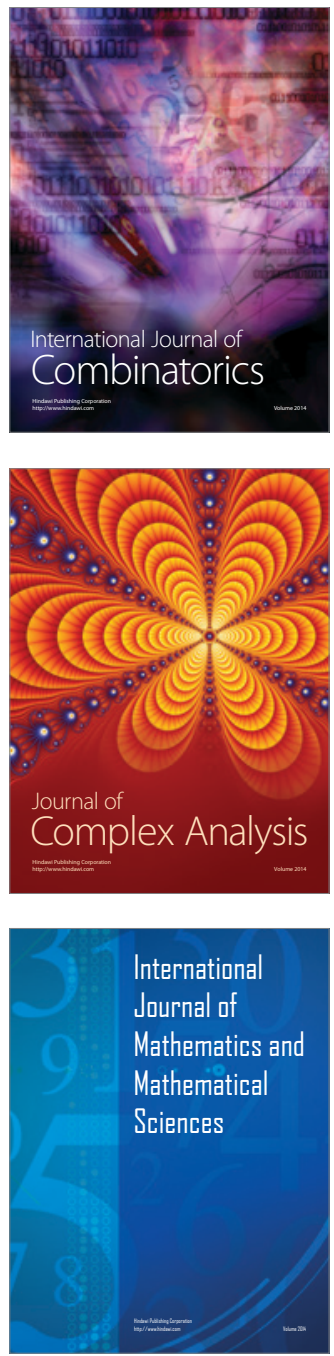
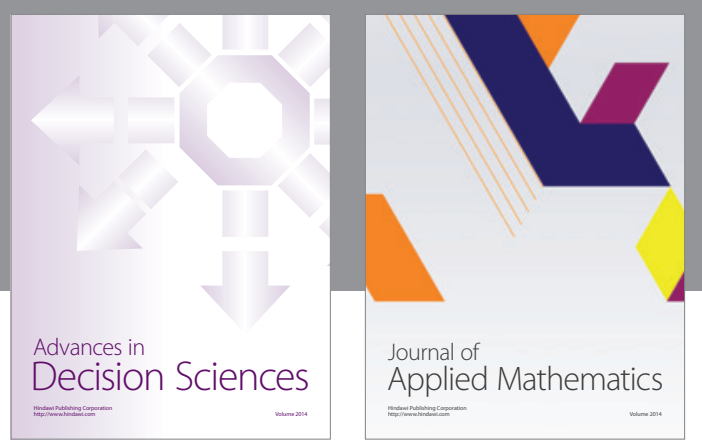

Algebra

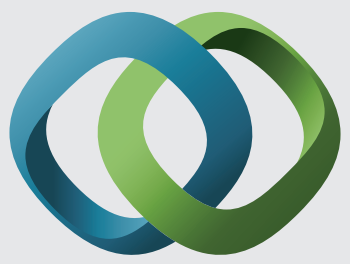

\section{Hindawi}

Submit your manuscripts at

http://www.hindawi.com
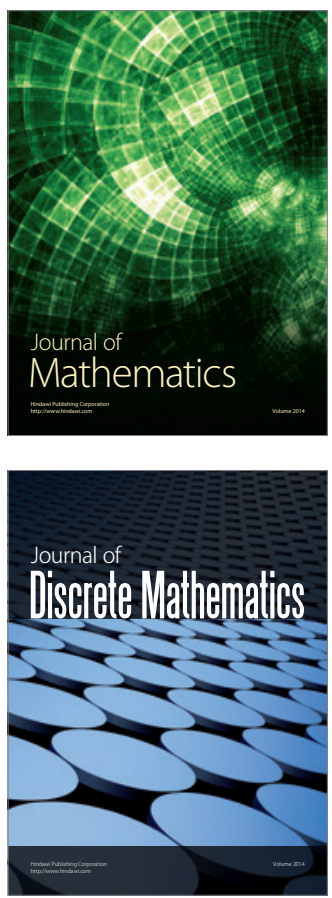

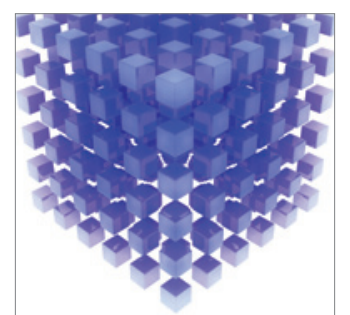

Mathematical Problems in Engineering
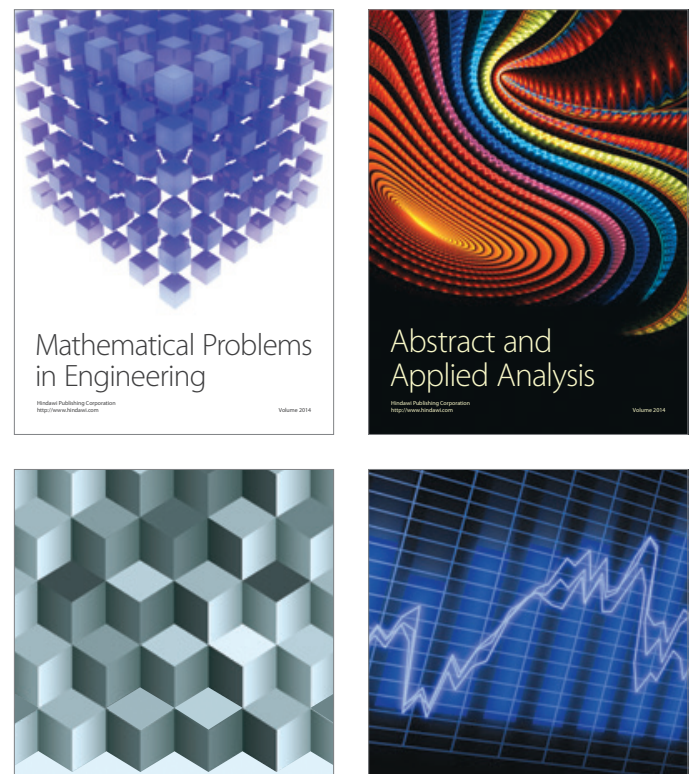

Journal of

Function Spaces

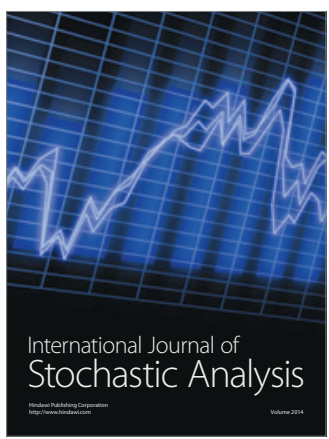

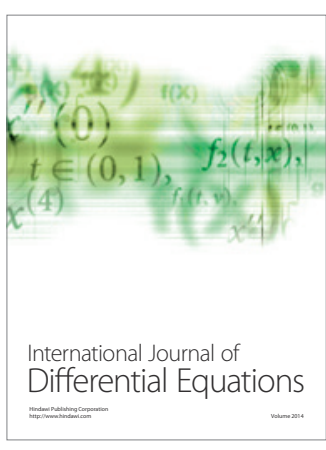
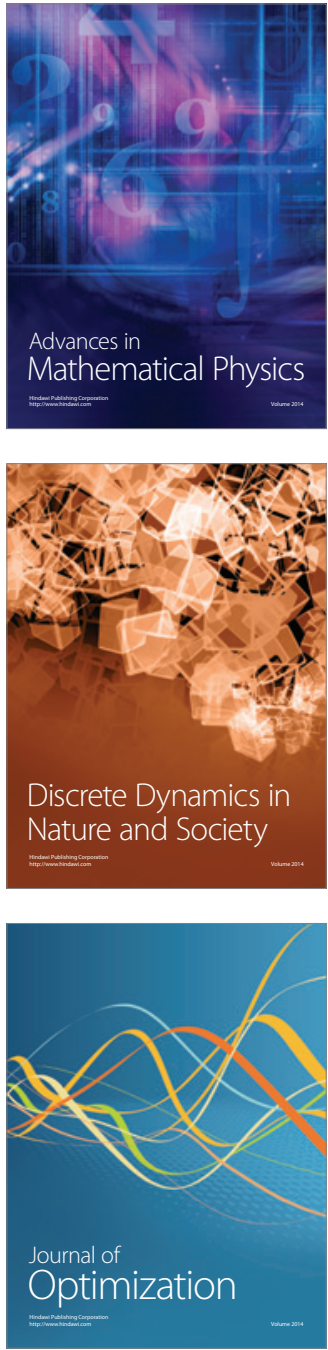